\title{
Sterically Bulky Thioureas as Air and Moisture Stable Ligands for Pd-Catalyzed Heck Reactions of Aryl Halides
}

\author{
Dan Yang, ${ }^{*}$ Ying-Chun Chen, and Nianyong Zhu
}

Department of Chemistry, The University of Hong Kong, Pokfulam Road, Hong Kong, China

yangdan@hku.hk

\section{Supporting Information}

1. Synthesis of acyclic thiourea ligands:

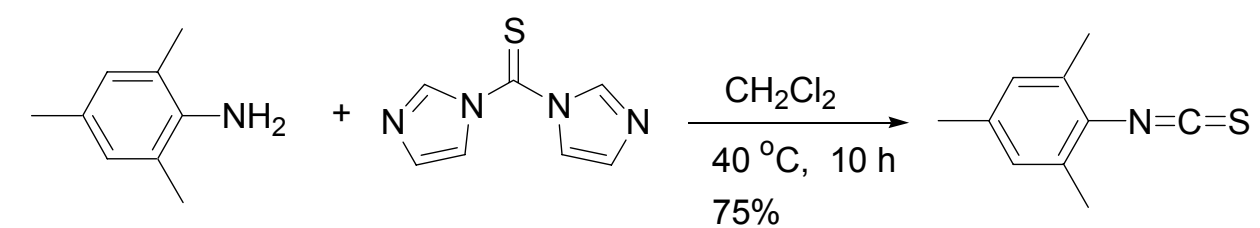

2

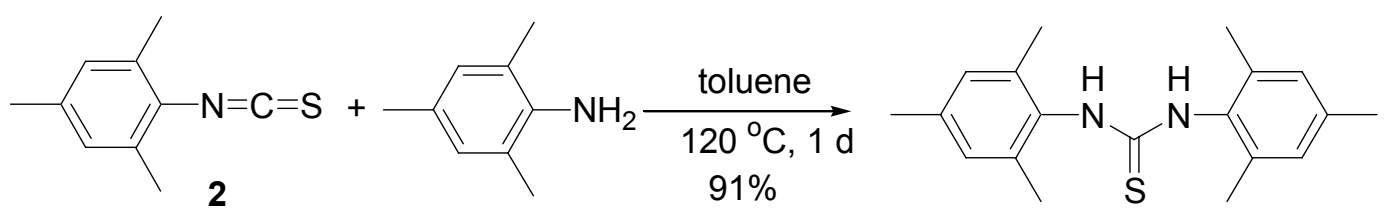

$1 \mathrm{~b}$

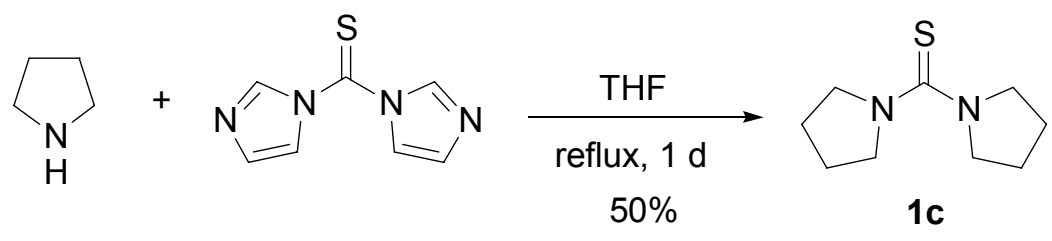

Scheme 1

\section{Preparation of 1b:}

2,4,6-Trimethylaniline $(0.16 \mathrm{~mL}, 1.2 \mathrm{mmol})$ and 1,1'-thiocarbonyl diimidazole $(210 \mathrm{mg}, 1.06$ 
mmol) were stirred in dichloromethane $(5 \mathrm{~mL})$ at $40{ }^{\circ} \mathrm{C}$ for $10 \mathrm{~h}$. 2-Mesityl isothiocyanate 2 was obtained (150 mg, 75\%) after flash chromatography on silica gel. ${ }^{1} \mathrm{H}$ NMR $\left(400 \mathrm{MHz}, \mathrm{CDCl}_{3}\right) \delta$ 6.84 (s, 2H), 2.32 (s, 6H), 2.26 (s, 3H); EI-MS: $177\left(\mathrm{M}^{+}, 100\right)$.

2-Mesityl isothiocyanate 2 (140 mg, $0.79 \mathrm{mmol}$ ) was heated with 2,4,6-trimethyl- aniline (128 $\mathrm{mg}, 0.95 \mathrm{mmol})$ in toluene $(1 \mathrm{~mL})$ at $120^{\circ} \mathrm{C}$ for one day, and $\mathrm{TLC}$ analysis indicated that all isothiocyanate had been consumed. Toluene was removed under vacuum, and the residue was recrystalized from hexane to give $\mathbf{1 b}$ as a white solid (223 mg, 91\%). M.p. 202-203 ${ }^{\circ} \mathrm{C} ;{ }^{1} \mathrm{H}$ NMR $\left(300 \mathrm{MHz}, \mathrm{CDCl}_{3}\right) \delta 7.84(\mathrm{~s}, 1 \mathrm{H}, \mathrm{NH}), 6.99(\mathrm{~s}, 2 \mathrm{H}), 6.85(\mathrm{~s}, 2 \mathrm{H}), 6.48(\mathrm{~s}, 1 \mathrm{H}, \mathrm{NH}), 2.38(\mathrm{~s}, 6 \mathrm{H})$, $2.30(\mathrm{~s}, 3 \mathrm{H}), 2.24$ (s, 3H), 2.19 (s, 6H); $\left.{ }^{13} \mathrm{C} \mathrm{NMR} \mathrm{(75} \mathrm{MHz,} \mathrm{CDCl}_{3}\right) \delta 181.3,139.1,137.7,137.2$, 136.0, 133.1, 130.5, 129.7, 129.0, 21.0, 18.5, 18.0; IR $\left(\mathrm{cm}^{-1}\right): 3405,3343,1511,1420,1245$; LRMS (EI): $312\left(\mathrm{M}^{+}, 44\right), 278$ (100); HRMS (EI): calcd for $\mathrm{C}_{19} \mathrm{H}_{24} \mathrm{~N}_{2} \mathrm{~S}$ 312.1660, found 312.1650 .

\section{Preparation of 1c:}

Pyrrolidine $(0.4 \mathrm{~g}, 5.6 \mathrm{mmol})$ and 1,1'-thiocarbonyl diimidazole $(0.3 \mathrm{~g}, 1.7 \mathrm{mmol})$ were refluxed in THF $(5 \mathrm{~mL})$ for $10 \mathrm{~h}$. The crude reaction mixture was concentrated, and flash chromatography on silica gel gave thiourea 1c as a white solid (160 mg, 50\%). M.p. 125-125.5 ${ }^{\circ} \mathrm{C} ;{ }^{1} \mathrm{H}$ NMR $\left(400 \mathrm{MHz}, \mathrm{CDCl}_{3}\right) \delta 3.62-3.59(\mathrm{~m}, 4 \mathrm{H}), 1.92-1.88(\mathrm{~m}, 4 \mathrm{H}) ;{ }^{13} \mathrm{C} \mathrm{NMR}(100 \mathrm{MHz}$, $\left.\mathrm{CDCl}_{3}\right) \delta 181.1,52.9,25.6$; IR $\left(\mathrm{cm}^{-1}\right):$ 1436, 1361, 1282, 1271; LRMS (EI): $184\left(\mathrm{M}^{+}, 100\right)$; HRMS (EI): calcd for $\mathrm{C}_{9} \mathrm{H}_{16} \mathrm{~N}_{2} \mathrm{~S} 184.1034$, found 184.1032 . 


\section{Synthesis of cyclic thioureas}

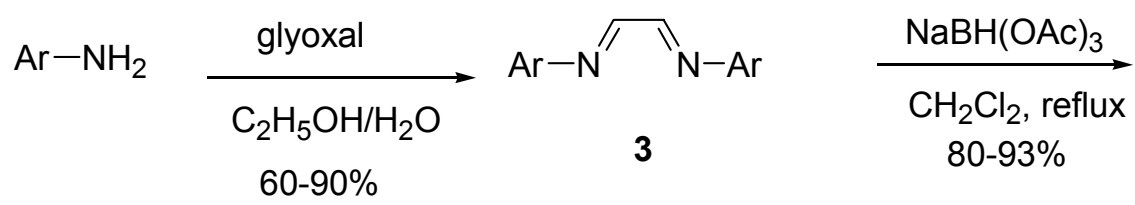

Method A 1,1'-Thiocarbonyldiimidazole
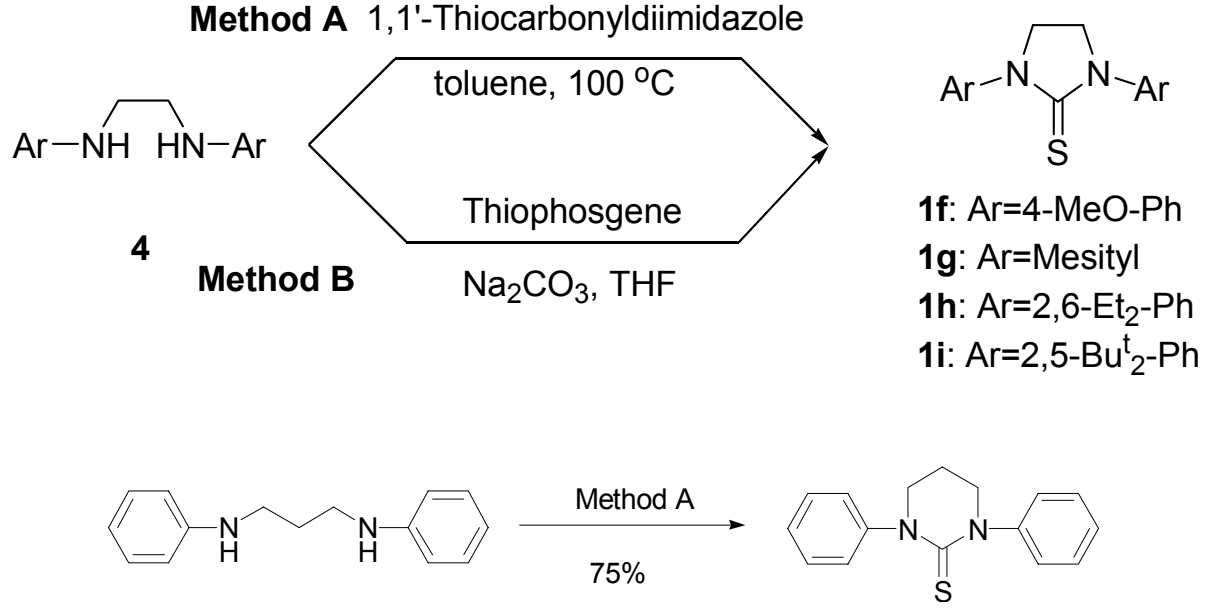

$1 \mathrm{j}$

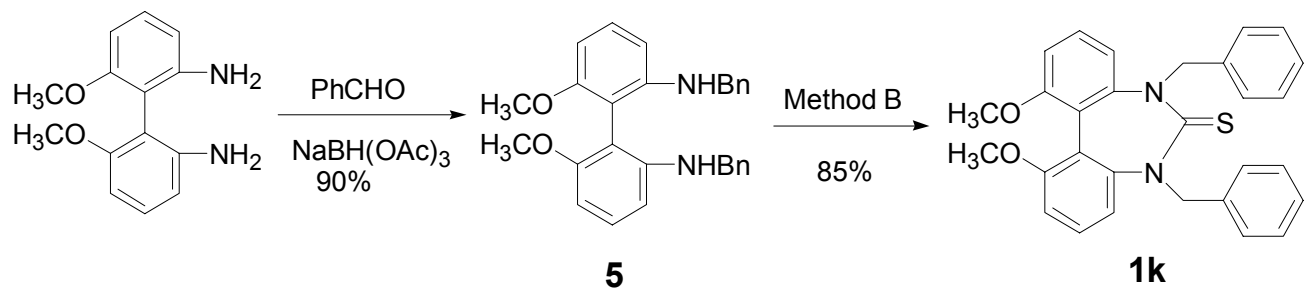

Scheme 2

The $N, N^{\prime}$-diaryl diamines were prepared following literature procedures. ${ }^{1}$ Two methods were used for the synthesis of cyclic thiourea ligands (Scheme 2).

\section{Method A:}

To a $N, N$ '-diaryl diamine solution in dry toluene was added 1,1 '-thiocarbonyl diimidazole (1.2 equiv). Then the solution was stirred at $100^{\circ} \mathrm{C}$ and the reaction was monitored by TLC. After completion, the solution was diluted with ethyl acetate and washed with dilute $\mathrm{HCl}$ and brine. The organic layer was concentrated under vacuum. The pure thiourea was obtained through flash chromatography or recrystallization from $95 \%$ ethanol. 


\section{Method B:}

To a stirred mixture of $N, N$ '-diaryl diamine and $\mathrm{Na}_{2} \mathrm{CO}_{3}$ (1.5 equiv) in dry THF was added a solution of thiophosgene (1.2 equiv) in THF dropwise at room temperature. After stirred at room temperature overnight, water and ethyl acetate were added. The organic layer was washed with dilute $\mathrm{HCl}$ and brine, dried and concentrated. The pure thiourea was obtained through flash chromatography or recrystallization from $95 \%$ ethanol.

\section{Preparation of 1f:}

Using method A; $75 \%$ yield. M.p. $167-168{ }^{\circ} \mathrm{C} ;{ }^{1} \mathrm{H}$ NMR $\left(300 \mathrm{MHz}, \mathrm{CDCl}_{3}\right) \delta 7.42(\mathrm{~d}, J=9.0$ $\mathrm{Hz}, 4 \mathrm{H}), 6.95(\mathrm{~d}, J=9.0 \mathrm{~Hz}, 4 \mathrm{H}), 4.08(\mathrm{~s}, 4 \mathrm{H}), 3.81(\mathrm{~s}, 6 \mathrm{H}) ;{ }^{13} \mathrm{C} \mathrm{NMR}\left(75 \mathrm{MHz}, \mathrm{CDCl}_{3}\right) \delta 182.2$, 158.1, 138.8, 127.5, 114.2, 55.4, 49.8; IR $\left(\mathrm{cm}^{-1}\right)$ : 1511, 1443, 1285; LRMS (EI): $314\left(\mathrm{M}^{+}, 100\right)$; HRMS (EI): calcd for $\mathrm{C}_{17} \mathrm{H}_{18} \mathrm{~N}_{2} \mathrm{O}_{2} \mathrm{~S}\left(\mathrm{M}^{+}\right)$314.1089, found 314.1088.

\section{Preparation of 1g:}

Using method B; 85\% yield. M.p. $218-218.5{ }^{\circ} \mathrm{C} ;{ }^{1} \mathrm{H}$ NMR $\left(400 \mathrm{MHz}, \mathrm{CDCl}_{3}\right) \delta 6.91$ (s, 4H), $3.94(\mathrm{~s}, 4 \mathrm{H}), 2.26(\mathrm{~s}, 6 \mathrm{H}), 2.24(\mathrm{~s}, 12 \mathrm{H}) ;{ }^{13} \mathrm{C} \mathrm{NMR}\left(75 \mathrm{MHz}, \mathrm{CDCl}_{3}\right) \delta 181.1,138.2,136.6$, 134.5, 129.5, 47.6, 21.1, 17.8; IR ( $\left.\mathrm{cm}^{-1}\right)$ : 1488, 1331, 1271; LRMS (FAB): $339\left(\mathrm{M}^{+}+1,100\right)$; HRMS (FAB): calcd for $\mathrm{C}_{21} \mathrm{H}_{26} \mathrm{~N}_{2} \mathrm{~S}\left(\mathrm{M}^{+}+1\right) 339.1894$, found 339.1879.

\section{Preparation of 1h:}

Using method B; 70\% yield. M.p. $152-153{ }^{\circ} \mathrm{C} ;{ }^{1} \mathrm{H}$ NMR $\left(300 \mathrm{MHz}, \mathrm{CDCl}_{3}\right) \delta 7.32(\mathrm{t}, J=6.6$ $\mathrm{Hz}, 2 \mathrm{H}), 7.20$ (d, J=7.5 Hz, 4H), $4.02(\mathrm{~s}, 4 \mathrm{H}), 2.80-2.70(\mathrm{~m}, 4 \mathrm{H}), 2.69-2.60(\mathrm{~m}, 4 \mathrm{H}), 1.33(\mathrm{t}, J$ $=7.5 \mathrm{~Hz}, 12 \mathrm{H}) ;{ }^{13} \mathrm{C} \mathrm{NMR}\left(75 \mathrm{MHz}, \mathrm{CDCl}_{3}\right) \delta 182.6,142.5,136.1,128.8,126.5,49.1,24.0,14.4$; IR (cm $\left.{ }^{-1}\right)$ : 1484, 1285; LRMS (EI): $366\left(\mathrm{M}^{+}, 39\right)$, 337 (100); HRMS (EI): calcd for $\mathrm{C}_{23} \mathrm{H}_{30} \mathrm{~N}_{2} \mathrm{~S}$ $\left(\mathrm{M}^{+}\right)$366.2130, found 366.2120.

\section{Preparation of 1i:}

Diimine 3i: 92\% yield. ${ }^{1} \mathrm{H}$ NMR (300 $\left.\mathrm{MHz} \mathrm{CDCl}_{3}\right) \delta 8.27(\mathrm{~s}, 2 \mathrm{H}), 7.35(\mathrm{~d}, J=8.3 \mathrm{~Hz}, 2 \mathrm{H})$, $7.25(\mathrm{~d}, J=8.3 \mathrm{~Hz}, 2 \mathrm{H}), 6.86(\mathrm{~s}, 2 \mathrm{H}), 1.43(\mathrm{~s}, 18 \mathrm{H}), 1.34(\mathrm{~s}, 18 \mathrm{H}) ;{ }^{13} \mathrm{C} \mathrm{NMR}\left(75 \mathrm{MHz}, \mathrm{CDCl}_{3}\right) \delta$ 158.6, 150.1, 150.0, 140.4, 126.0, 123.8, 116.0, 35.3, 34.4, 31.3, 30.5; IR $\left(\mathrm{cm}^{-1}\right)$ : 1609, 1492 , 1265; LRMS (EI): $432\left(\mathrm{M}^{+}, 100\right)$; $\operatorname{HRMS}(\mathrm{EI})$ : calcd for $\mathrm{C}_{30} \mathrm{H}_{44} \mathrm{~N}_{2}\left(\mathrm{M}^{+}\right)$432.3504, found 
432.3504 .

Diamine 4i: 90\% yield. ${ }^{1} \mathrm{H}$ NMR (300 MHz, $\left.\mathrm{CDCl}_{3}\right) \delta 7.18(\mathrm{~d}, J=6.1 \mathrm{~Hz}, 2 \mathrm{H}), 6.80(\mathrm{~s}, 2 \mathrm{H})$, $6.75(\mathrm{~d}, J=6.1 \mathrm{~Hz}, 2 \mathrm{H}), 4.18(\mathrm{br} \mathrm{s}, 2 \mathrm{H}, \mathrm{NH}), 3.57$ (s, 4H), 1.39 (s, 18H), $1.32(\mathrm{~s}, 18 \mathrm{H}) ;{ }^{13} \mathrm{C}$ $\operatorname{NMR}\left(75 \mathrm{MHz}, \mathrm{CDCl}_{3}\right) \delta 149.9,146.2,131.2,126.0,114.6,110.0,45.0,34.4,33.8,31.4,30.2$; IR $\left(\mathrm{cm}^{-1}\right)$ : 3688, 3601, 1561, 1265; LRMS (EI): 436 (M+, 20), 219 (100); HRMS (EI): calcd for $\mathrm{C}_{30} \mathrm{H}_{48} \mathrm{~N}_{2}\left(\mathrm{M}^{+}\right)$436.3817, found 436.3817 .

Thiourea li was prepared using method B. A solution of Thiophosgene in dilute THF must be dropped very slowly. 1i was isolated as a white solid (75\% yield) after flash chromatography on silica gel. M.p. $212-214{ }^{\circ} \mathrm{C} ;{ }^{1} \mathrm{H}$ NMR $\left(400 \mathrm{MHz}, \mathrm{CDCl}_{3}\right) \delta 7.45(\mathrm{~d}, J=8.5 \mathrm{~Hz}, 2 \mathrm{H}), 7.32(\mathrm{~d}, J=$ $8.5 \mathrm{~Hz}, 2 \mathrm{H}), 7.02(\mathrm{~s}, 2 \mathrm{H}), 4.06-4.03(\mathrm{~m}, 2 \mathrm{H}), 3.93-3.91(\mathrm{~m}, 2 \mathrm{H}), 1.50(\mathrm{~s}, 18 \mathrm{H}), 1.30(\mathrm{~s}, 18 \mathrm{H})$; ${ }^{13} \mathrm{C}$ NMR $\left(100 \mathrm{MHz}, \mathrm{CDCl}_{3}\right) \delta 183.5,150.4,145.0,140.8,128.0,127.8,125.3,53.4,35.4,34.3$, 32.1, 31.3; IR $\left(\mathrm{cm}^{-1}\right)$ : 1418, 1275; LRMS (FAB): $479\left(\mathrm{M}^{+}+\mathrm{H}\right)$; FAB-HRMS: calcd for $\mathrm{C}_{31} \mathrm{H}_{46} \mathrm{~N}_{2} \mathrm{~S}\left(\mathrm{M}^{+}+\mathrm{H}\right) 479.3460$, found 479.3460 .

\section{Preparation of $1 \mathrm{j}$ :}

Using method A, 75\% yield. M.p. $173-174{ }^{\circ} \mathrm{C} ;{ }^{1} \mathrm{H}$ NMR $\left(300 \mathrm{MHz}, \mathrm{CDCl}_{3}\right) \delta$ 7.41-7.15 (m, 10H), 3.82-3.77 (m, 4H), 2.32-2.24 (m, 2H); $\left.{ }^{13} \mathrm{C} \mathrm{NMR} \mathrm{(75} \mathrm{MHz,} \mathrm{CDCl}_{3}\right) \delta$ 180.7, 147.4, 129.2, 127.4, 125.8, 51.4, 22.3; IR ( $\left.\mathrm{cm}^{-1}\right)$ : 1494, 1285; LRMS (EI): 268 (M+13); EI-HRMS: calcd for $\mathrm{C}_{16} \mathrm{H}_{16} \mathrm{~N}_{2} \mathrm{~S}\left(\mathrm{M}^{+}\right)$268.1034, found 268.1015.

\section{Preparation of $1 \mathrm{k}$ :}

To a stirred suspension of racemic 2,2'-diamino-6,6'-dimethoxy-biphenyl ${ }^{2}$ (60mg, $0.25 \mathrm{mmol}$ ) and $\mathrm{NaBH}(\mathrm{OAc})_{3}(212 \mathrm{mg}, 1 \mathrm{mmol})$ in dichloromethane $(10 \mathrm{~mL})$ was added a solution of benzaldehyde $(0.06 \mathrm{ml}, 0.58 \mathrm{mmol})$ in dichloromethane $(2 \mathrm{~mL})$ dropwise at room temperature. Then the mixture was stirred overnight. Flash chromatography on silica gel gave $N, N$ '-dibenzyl diamine 5 as a white solid (94 mg, 90\%). ${ }^{1} \mathrm{H}$ NMR $\left(300 \mathrm{MHz}, \mathrm{CDCl}_{3}\right) \delta 7.26-7.11(\mathrm{~m}, 12 \mathrm{H})$, $6.38(\mathrm{~d}, J=8.2 \mathrm{~Hz}, 2 \mathrm{H}), 6.32$ (d, $J=7.7 \mathrm{~Hz}, 2 \mathrm{H}), 4.32$ (s, $4 \mathrm{H}), 4.17$ (br s $2 \mathrm{H}), 3.70(\mathrm{~s}, 6 \mathrm{H}) ;{ }^{13} \mathrm{C}$ NMR $\left(75 \mathrm{MHz}, \mathrm{CDCl}_{3}\right) \delta 158.1,147.3,139.9,129.6,128.4,126.7,126.6,107.2,104.2,100.6$, 55.7, 47.5; IR ( $\left.\mathrm{cm}^{-1}\right): 3432,3086,3051,2938,1586,496,1472,1422,1282$, 1131; LRMS (EI): 424 ( $\left.\mathrm{M}^{+}, 33\right), 333$ (100); HRMS (EI): calcd for $\mathrm{C}_{28} \mathrm{H}_{28} \mathrm{~N}_{2} \mathrm{O}_{2} \mathrm{~S}\left(\mathrm{M}^{+}\right) 424.2151$, found 424.2138.

Thiourea 1k was prepared using method B, 85\% yield. M.p. $179-180{ }^{\circ} \mathrm{C} ;{ }^{1} \mathrm{H}$ NMR $(400 \mathrm{MHz}$, 
$\left.\mathrm{CDCl}_{3}\right) \delta 7.27(\mathrm{t}, J=8.2 \mathrm{~Hz}, 2 \mathrm{H}), 7.04-7.00(\mathrm{~m}, 6 \mathrm{H}), 6.88(\mathrm{~d}, J=8.2 \mathrm{~Hz}, 2 \mathrm{H}), 6.83-6.80(\mathrm{~m}$, $6 \mathrm{H}), 5.72(\mathrm{~d}, J=15.3 \mathrm{~Hz}, 2 \mathrm{H}), 4.81(\mathrm{~d}, J=15.3 \mathrm{~Hz}, 2 \mathrm{H}), 3.75(\mathrm{~s}, 6 \mathrm{H}) ;{ }^{13} \mathrm{C} \mathrm{NMR}(75 \mathrm{MHz}$, $\left.\mathrm{CDCl}_{3}\right) \delta 199.6,157.2,147.7,137.1,128.7,127.9,127.5,126.7,121.8,113.9,108.8,56.8,55.9$; IR $\left(\mathrm{cm}^{-1}\right): 3051,1592,1579,1464,1420,1245,1190$; LRMS (EI): $466\left(\mathrm{M}^{+}, 100\right), 375$ (86); HRMS (EI): calcd for $\mathrm{C}_{29} \mathrm{H}_{26} \mathrm{~N}_{2} \mathrm{O}_{2} \mathrm{~S}\left(\mathrm{M}^{+}\right)$466.1715, found 466.1718.

\section{General procedure for Heck reaction of aryl iodides and olefins}

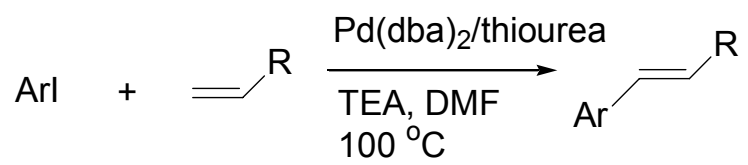

$\mathrm{Pd}(\mathrm{dba})_{2}(1.5 \mathrm{mg}, 0.0025 \mathrm{mmol})$ and thiourea (4 equiv) were stirred in DMF $(0.5 \mathrm{~mL})$ for 0.5 $\mathrm{h}$ at rt. Iodobenzene $(0.28 \mathrm{~mL}, 2.5 \mathrm{mmol}$, substrate/catalyst ratio $=1000: 1)$ and methyl acrylate $(0.27 \mathrm{~mL}, 3.0 \mathrm{mmol})$ and TEA $(0.42 \mathrm{~mL}, 3.0 \mathrm{mmol})$ were then added. The flask was sealed with rubber septa and heated at $100^{\circ} \mathrm{C}$ (the same result was obtained when the reaction was conducted with a condenser in open air). After the indicated time, the solution was diluted with ethyl acetate $(20 \mathrm{~mL})$ and washed with water and brine. Ethyl acetate was removed under vacuum and nitrobenzene $(0.128 \mathrm{~mL})$ was added as an internal standard. The yield of coupling product was determined by ${ }^{1} \mathrm{H}$ NMR (400 MHz or $300 \mathrm{MHz}$ ) analysis, by comparing the peak intensities of the $\alpha / \beta-\mathrm{H}$ of the product and the ortho-H of nitrobenzene (internal standard).

When the reactions were conducted at a lower catalyst loading, the calculated amount of the solution of $\mathrm{Pd}(\mathrm{dba})_{2}$ and thiourea in $\mathrm{DMF}(0.0025 \mathrm{M})$ was used.

${ }^{\mathrm{Ph}}{ }^{\mathrm{COOMe}} \mathrm{H}$ NMR $\left(300 \mathrm{MHz}, \mathrm{CDCl}_{3}\right) \delta 7.67-7.63(\mathrm{~m}, 2 \mathrm{H}), 7.54(\mathrm{~d}, J=4.1 \mathrm{~Hz}, 2 \mathrm{H}), 7.38(\mathrm{~d}$, $J=3.3 \mathrm{~Hz}, 1 \mathrm{H}), 7.10(\mathrm{t}, J=6.5 \mathrm{~Hz}, 1 \mathrm{H}), 6.44(\mathrm{~d}, J=16.1 \mathrm{~Hz}, 1 \mathrm{H}), 3.81(\mathrm{~s}, 3 \mathrm{H})$. To determine the reaction yield, the product peak at $6.44 \mathrm{ppm}$ was selected for comparison with that of the ortho-H (at $8.20 \mathrm{ppm}$ ) of nitrobenzene (internal standard).

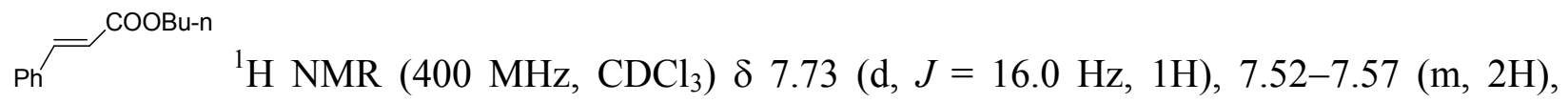
$7.40-7.45(\mathrm{~m}, 3 \mathrm{H}), 6.49(\mathrm{~d}, J=16.0 \mathrm{~Hz}, 1 \mathrm{H}), 4.26(\mathrm{t}, J=6.9 \mathrm{~Hz}, 2 \mathrm{H}), 1.71-1.78(\mathrm{~m}, 2 \mathrm{H})$, $1.54-1.45(\mathrm{~m}, 2 \mathrm{H}), 1.00(\mathrm{t}, J=7.4 \mathrm{~Hz}, 3 \mathrm{H})$.

$\mathrm{Ph} \approx$ COOBu-t ${ }^{1} \mathrm{H}$ NMR $\left(300 \mathrm{MHz}, \mathrm{CDCl}_{3}\right) \delta 7.73(\mathrm{~d}, J=16.0 \mathrm{~Hz}, 1 \mathrm{H}), 7.53-7.57(\mathrm{~m}, 2 \mathrm{H})$, 
7.40-7.45 (m, 3H), $6.49(\mathrm{~d}, J=16.0 \mathrm{~Hz}, 1 \mathrm{H}), 1.34(\mathrm{~s}, 9 \mathrm{H})$.

${ }_{\mathrm{Ph}}{ }^{\mathrm{Ph}}{ }^{1} \mathrm{H}$ NMR $\left(300 \mathrm{MHz}, \mathrm{CDCl}_{3}\right) \delta 7.53(\mathrm{~d}, J=7.2 \mathrm{~Hz}, 4 \mathrm{H}), 7.38(\mathrm{dd}, J=7.1,1.5 \mathrm{~Hz}, 4 \mathrm{H})$, $7.28(\mathrm{~d}, J=7.2 \mathrm{~Hz}, 2 \mathrm{H}), 7.13(\mathrm{~s}, 2 \mathrm{H})$.

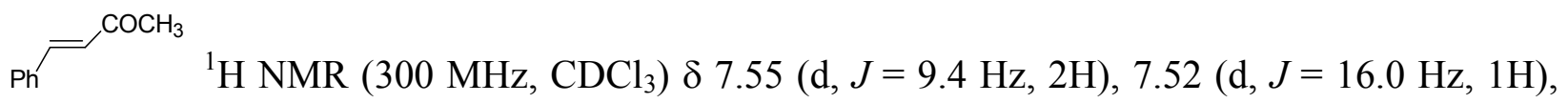
$7.40(\mathrm{t}, J=3.5 \mathrm{~Hz}, 3 \mathrm{H}), 6.72(\mathrm{~d}, J=16.0 \mathrm{~Hz}, 1 \mathrm{H}), 2.39$ (s, 3H).

${ }^{1} \mathrm{H} \mathrm{NMR}\left(300 \mathrm{MHz}, \mathrm{CDCl}_{3}\right) \delta 7.63(\mathrm{~d}, J=16.2 \mathrm{~Hz}, 1 \mathrm{H}), 7.43(\mathrm{~d}, J=6.2 \mathrm{~Hz}$, 2H), $7.35(\mathrm{~d}, J=6.2 \mathrm{~Hz}, 2 \mathrm{H}), 6.40(\mathrm{~d}, J=16.2 \mathrm{~Hz}, 1 \mathrm{H}), 4.26(\mathrm{t}, J=6.9 \mathrm{~Hz}, 2 \mathrm{H}), 1.78-1.71(\mathrm{~m}$, 2H), 1.54-1.45 (m, 2H), $1.00(\mathrm{t}, J=7.4 \mathrm{~Hz}, 3 \mathrm{H})$.

${ }^{1} \mathrm{H} \mathrm{NMR}\left(400 \mathrm{MHz}, \mathrm{CDCl}_{3}\right) \delta 7.68(\mathrm{~d}, J=16.0 \mathrm{~Hz}, 1 \mathrm{H}), 7.51(\mathrm{~d}, J=8.9$ $\mathrm{Hz}, 2 \mathrm{H}), 6.94(\mathrm{~d}, J=8.9 \mathrm{~Hz}, 2 \mathrm{H}), 6.36(\mathrm{~d}, J=16.0 \mathrm{~Hz}, 1 \mathrm{H}), 4.25(\mathrm{t}, J=6.8 \mathrm{~Hz}, 2 \mathrm{H}), 3.87(\mathrm{~s}, 3 \mathrm{H})$, $1.76-1.70(\mathrm{~m}, 2 \mathrm{H}), 1.52-1.46(\mathrm{~m}, 2 \mathrm{H}), 1.02(\mathrm{t}, J=7.5 \mathrm{~Hz}, 3 \mathrm{H})$.

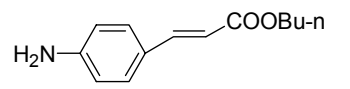

${ }^{1} \mathrm{H}$ NMR $\left(400 \mathrm{MHz}, \mathrm{CDCl}_{3}\right) \delta 7.70(\mathrm{~d}, J=8.4 \mathrm{~Hz}, 2 \mathrm{H}), 7.56(\mathrm{~d}, J=15.7$ $\mathrm{Hz}, 1 \mathrm{H}), 6.62(\mathrm{~d}, J=8.4 \mathrm{~Hz}, 2 \mathrm{H}), 6.51(\mathrm{~d}, J=15.7 \mathrm{~Hz}, 1 \mathrm{H}), 6.17(\mathrm{~s}, 2 \mathrm{H}), 4.26(\mathrm{t}, J=6.9 \mathrm{~Hz}, 2 \mathrm{H})$, $1.78-1.77(\mathrm{~m}, 2 \mathrm{H}), 1.54-1.45(\mathrm{~m}, 2 \mathrm{H}), 1.00(\mathrm{t}, J=7.4 \mathrm{~Hz}, 3 \mathrm{H})$.

${ }_{\mathrm{Ph}}{ }^{\mathrm{COOMe}}{ }^{1} \mathrm{H}$ NMR $\left(300 \mathrm{MHz}, \mathrm{CDCl}_{3}\right) \delta 7.55(\mathrm{~d}, J=6.9 \mathrm{~Hz}, 2 \mathrm{H}), 7.40-7.19(\mathrm{~m}, 4 \mathrm{H}), 3.82(\mathrm{~s}$, $3 \mathrm{H}), 2.13(\mathrm{~s}, 3 \mathrm{H})$.

${ }_{\mathrm{Ph}}{ }^{1} \mathrm{H}$ NMR $\left(300 \mathrm{MHz}, \mathrm{CDCl}_{3}\right) \delta 7.53-7.45(\mathrm{~m}, 3 \mathrm{H}), 7.37-7.35(\mathrm{~m}, 2 \mathrm{H}), 6.13(\mathrm{q}, J=$ $1.2 \mathrm{~Hz}, 1 \mathrm{H}), 3.75$ (s, 3H), 2.58 (d, $J=1.3 \mathrm{~Hz}, 3 \mathrm{H})$.

\section{General procedure for Heck reaction of aryl bromides and olefins}

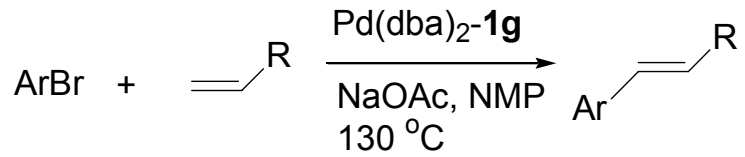

$\operatorname{Pd}(\mathrm{dba})_{2}(1.5 \mathrm{mg}, 0.0025 \mathrm{mmol})$ and thiourea $1 \mathrm{~g}(3.4 \mathrm{mg}, 0.01 \mathrm{mmol})$ were stirred in NMP $(0.5 \mathrm{~mL})$ for $0.5 \mathrm{~h}$ at $\mathrm{rt}$. Aryl bromide $(2.5 \mathrm{mmol}, S / C=1000)$, olefin $(3.8 \mathrm{mmol})$ and sodium acetate $330 \mathrm{mg}(3.8 \mathrm{mmol})$ were added in turn. Then the flask was sealed with a septa and heated at $130^{\circ} \mathrm{C}$. After indicated time, the solution was dilute with ethyl acetate $(20 \mathrm{~mL})$ and washed 
with water and brine. Ethyl acetate was removed under vacuum and nitrobenzene $(0.128 \mathrm{~mL})$ was added as internal standard. The yield of coupling product was determined by ${ }^{1} \mathrm{H}$ NMR (400 $\mathrm{MHz}$ or $300 \mathrm{MHz}$ ) analysis, by comparing the peak intensities of the $\alpha / \beta-\mathrm{H}$ of the product and the ortho-H of nitrobenzene (internal standard).

${ }^{1} \mathrm{H} \mathrm{NMR}\left(300 \mathrm{MHz}, \mathrm{CDCl}_{3}\right) \delta 9.99(\mathrm{~s}, 1 \mathrm{H}), 7.87(\mathrm{~d}, J=8.1 \mathrm{~Hz}, 2 \mathrm{H})$, 7.70-7.62 (m, 3H), $6.52(\mathrm{~d}, J=15.9 \mathrm{~Hz}, 1 \mathrm{H}), 3.79(\mathrm{~s}, 3 \mathrm{H})$.

${ }^{1} \mathrm{H}$ NMR $\left(300 \mathrm{MHz} \mathrm{CDCl}_{3}\right) \delta 7.80-7.75(\mathrm{~m}, 3 \mathrm{H}), 7.42(\mathrm{~d}, J=6.8 \mathrm{~Hz}$, 2H), $6.34(\mathrm{~d}, J=16.1 \mathrm{~Hz}, 1 \mathrm{H}), 3.63(\mathrm{~s}, 3 \mathrm{H}), 2.42(\mathrm{~s}, 3 \mathrm{H})$.

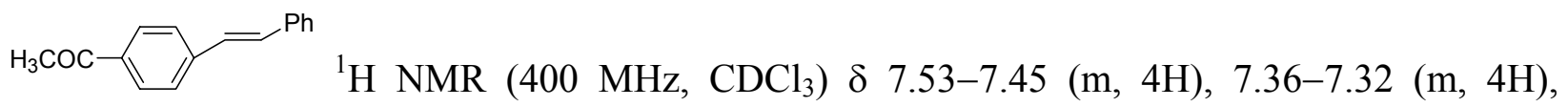
7.28-7.26 (m, 2H), 7.17 (d, $J=12.3 \mathrm{~Hz}, 1 \mathrm{H}), 7.07$ (d, $J=12.3 \mathrm{~Hz}, 1 \mathrm{H}), 2.55$ (s, 3H).

${ }^{1} \mathrm{H}$ NMR $\left(300 \mathrm{MHz}, \mathrm{CDCl}_{3}\right) \delta 7.85-7.32(\mathrm{~m}, 15 \mathrm{H}), 6.24(\mathrm{~d}, J=16.2 \mathrm{~Hz}, 1 \mathrm{H})$.

${ }^{\mathrm{N}=}{ }^{\mathrm{Ph}} \mathrm{H} \mathrm{NMR}\left(300 \mathrm{MHz}, \mathrm{CDCl}_{3}\right) \delta 8.70(\mathrm{~d}, J=1.3 \mathrm{~Hz}, 1 \mathrm{H}), 8.45(\mathrm{~d}, J=3.5 \mathrm{~Hz}, 1 \mathrm{H}), 7.52$ $(\mathrm{d}, J=9.0 \mathrm{~Hz}, 1 \mathrm{H}), 7.36-7.33(\mathrm{~m}, 2 \mathrm{H}), 7.30-7.25(\mathrm{~m}, 4 \mathrm{H}), 7.10(\mathrm{~d}, J=16.2 \mathrm{~Hz}, 1 \mathrm{H}), 7.00(\mathrm{~d}, J$ $=16.2 \mathrm{~Hz}, 1 \mathrm{H})$.

General procedure for Heck reaction of deactivated aryl bromides and activated chlorides with olefins $^{3}$

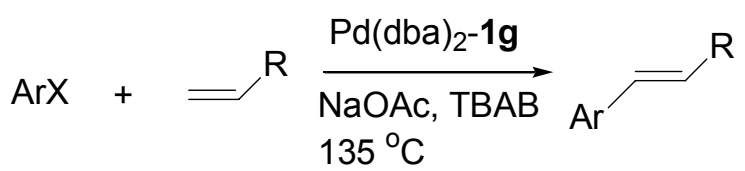

$\mathrm{Pd}(\mathrm{dba})_{2}(1.5 \mathrm{mg}, 0.0025 \mathrm{mmol})$, thiourea $1 \mathrm{~g}(3.4 \mathrm{mg}, 0.01 \mathrm{mmol})$ and sodium acetate $(33 \mathrm{mg}$, $3.8 \mathrm{mmol})$ were stirred in molten $\operatorname{TBAB}(0.5 \mathrm{~g})$ for $10 \mathrm{~min}$ at $100^{\circ} \mathrm{C}$. Aryl halide $(0.25 \mathrm{mmol}$, $S / C=100)$ and olefin $(0.38 \mathrm{mmol})$ were added in turn. Then the flask was sealed with a septa and heated at $135^{\circ} \mathrm{C}$. After indicated time, the solution was dilute with ethyl acetate $(20 \mathrm{~mL})$ and washed with water and brine. Ethyl acetate was removed under vacuum and nitrobenzene $(0.0128 \mathrm{~mL})$ was added as internal standard. The yield of coupling product was determined by ${ }^{1} \mathrm{H}$ NMR (400 MHz or $300 \mathrm{MHz}$ ) analysis, by comparing the peak intensities of the $\alpha / \beta-\mathrm{H}$ of the product and the ortho-H of nitrobenzene (internal standard). 


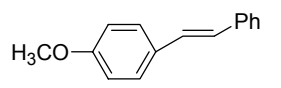

${ }^{1} \mathrm{H}$ NMR (400 MHz, $\left.\mathrm{CDCl}_{3}\right) \delta$ 7.64-7.52 (m, 4H), 7.45-7.40 (m, 3H), 7.33 (d, $J$

$=12.1 \mathrm{~Hz}, 1 \mathrm{H}), 7.10(\mathrm{~d}, J=12.1 \mathrm{~Hz}, 1 \mathrm{H}), 6.98(\mathrm{~d}, J=8.2 \mathrm{~Hz}, 2 \mathrm{H}), 3.88(\mathrm{~s}, 3 \mathrm{H})$.

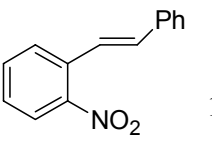

${ }^{1} \mathrm{H}$ NMR $\left(400 \mathrm{MHz}, \mathrm{CDCl}_{3}\right) \delta 7.93(\mathrm{~d}, J=7.0 \mathrm{~Hz}, 1 \mathrm{H}), 7.74(\mathrm{~d}, J=7.0 \mathrm{~Hz}, 1 \mathrm{H})$, 7.60-7.51 (m, 5H), 7.39-7.30 (m, 3H), 7.07 (d, $J=16.1 \mathrm{~Hz}, 1 \mathrm{H})$.

${ }^{1} \mathrm{H}$ NMR $\left(400 \mathrm{MHz}, \mathrm{CDCl}_{3}\right) \delta 8.13(\mathrm{~d}, J=17.3 \mathrm{~Hz}, 1 \mathrm{H}), 8.05(\mathrm{~d}, J=7.8 \mathrm{~Hz}, 1 \mathrm{H})$, $7.84(\mathrm{~d}, J=6.8 \mathrm{~Hz}, 1 \mathrm{H}), 7.27-7.24(\mathrm{~m}, 2 \mathrm{H}), 6.36(\mathrm{~d}, J=17.3 \mathrm{~Hz}, 1 \mathrm{H}), 4.22(\mathrm{t}, J=5.0 \mathrm{~Hz}, 2 \mathrm{H})$, $1.71-1.67(\mathrm{~m}, 2 \mathrm{H}), 1.32-1.28(\mathrm{~m}, 2 \mathrm{H}), 0.96(\mathrm{t}, J=6.8 \mathrm{~Hz}, 3 \mathrm{H})$.

${ }^{1} \mathrm{H} \mathrm{NMR}\left(300 \mathrm{MHz}, \mathrm{CDCl}_{3}\right) \delta 7.62(\mathrm{~d}, J=15.6 \mathrm{~Hz}, 1 \mathrm{H}), 7.41(\mathrm{~d}, J=7.1$ $\mathrm{Hz}, 2 \mathrm{H}), 6.66(\mathrm{~d}, J=7.1 \mathrm{~Hz}, 2 \mathrm{H}), 6.22(\mathrm{~d}, J=15.6 \mathrm{~Hz}, 1 \mathrm{H}), 4.18(\mathrm{t}, J=6.7 \mathrm{~Hz}, 2 \mathrm{H}), 3.00(\mathrm{~s}, 6 \mathrm{H})$, $1.71-1.66(\mathrm{~m}, 2 \mathrm{H}), 1.47-1.40(\mathrm{~m}, 2 \mathrm{H}), 0.96(\mathrm{t}, J=8.2 \mathrm{~Hz}, 3 \mathrm{H})$.

\section{References}

1. (a) Arduengo, A. J.; Krafczyk, R.; Schmutzler, R. Tetrahedron 1999, 55, 14523. (b) Lee, S.; Hartwig, J. F. J. Org. Chem. 2001, 66, 3402.

2. Chen, Y.-X.; Li, Y.-M.; Lam, K.-H.; Chan, A. S. C. Chin. J. Chem. 2001, 19, 794.

3. Selvakumar, K.; Zapf, A.; Beller, M. Org. Lett. 2002, 4, 3031. 


\section{$\mathrm{X}$-Ray Structures of Cis- and Trans- $\mathrm{PdCl}_{2} \cdot(1 \mathrm{~g})_{2}$}

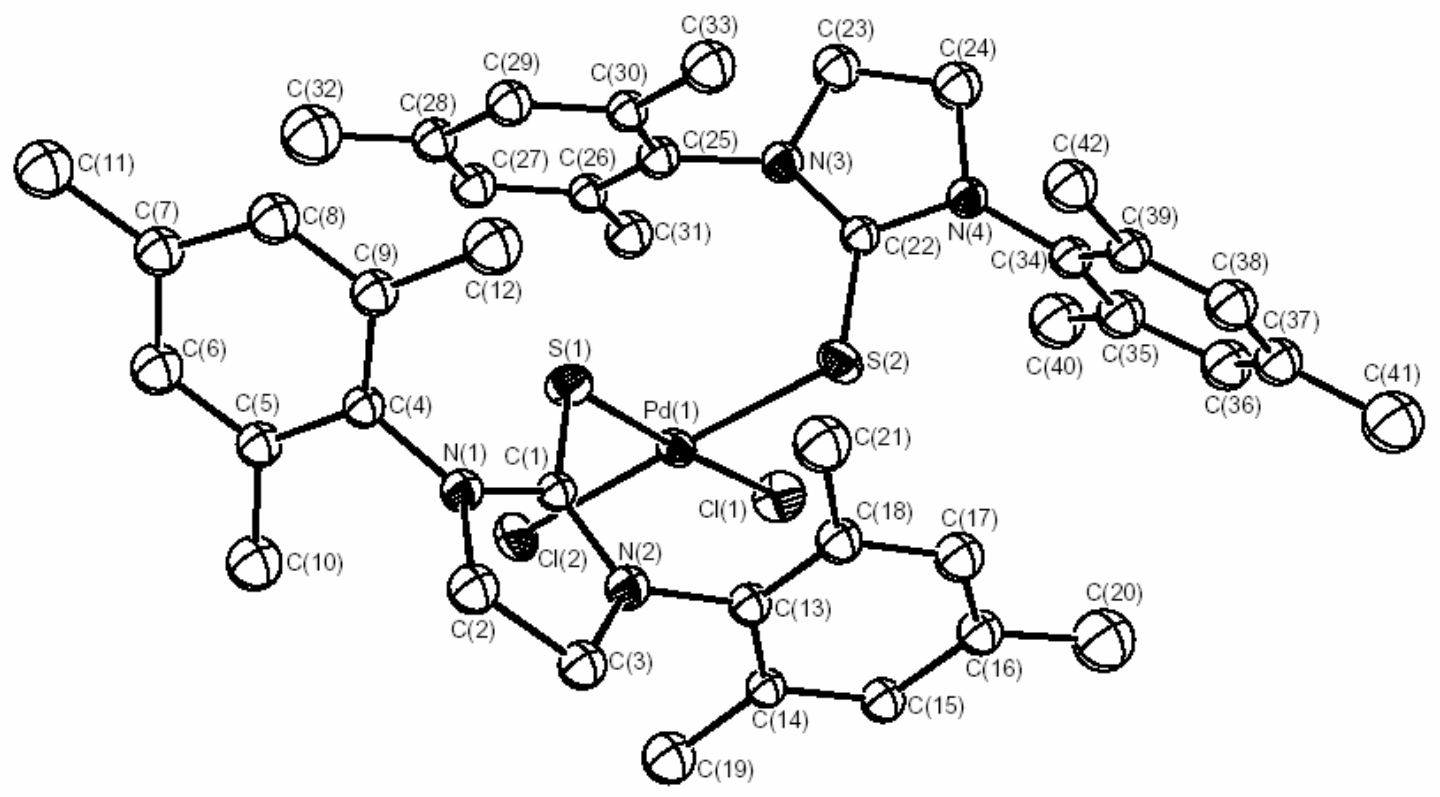

Cis- $\mathrm{PdCl}_{2} \cdot(\mathbf{1 g})_{2}$

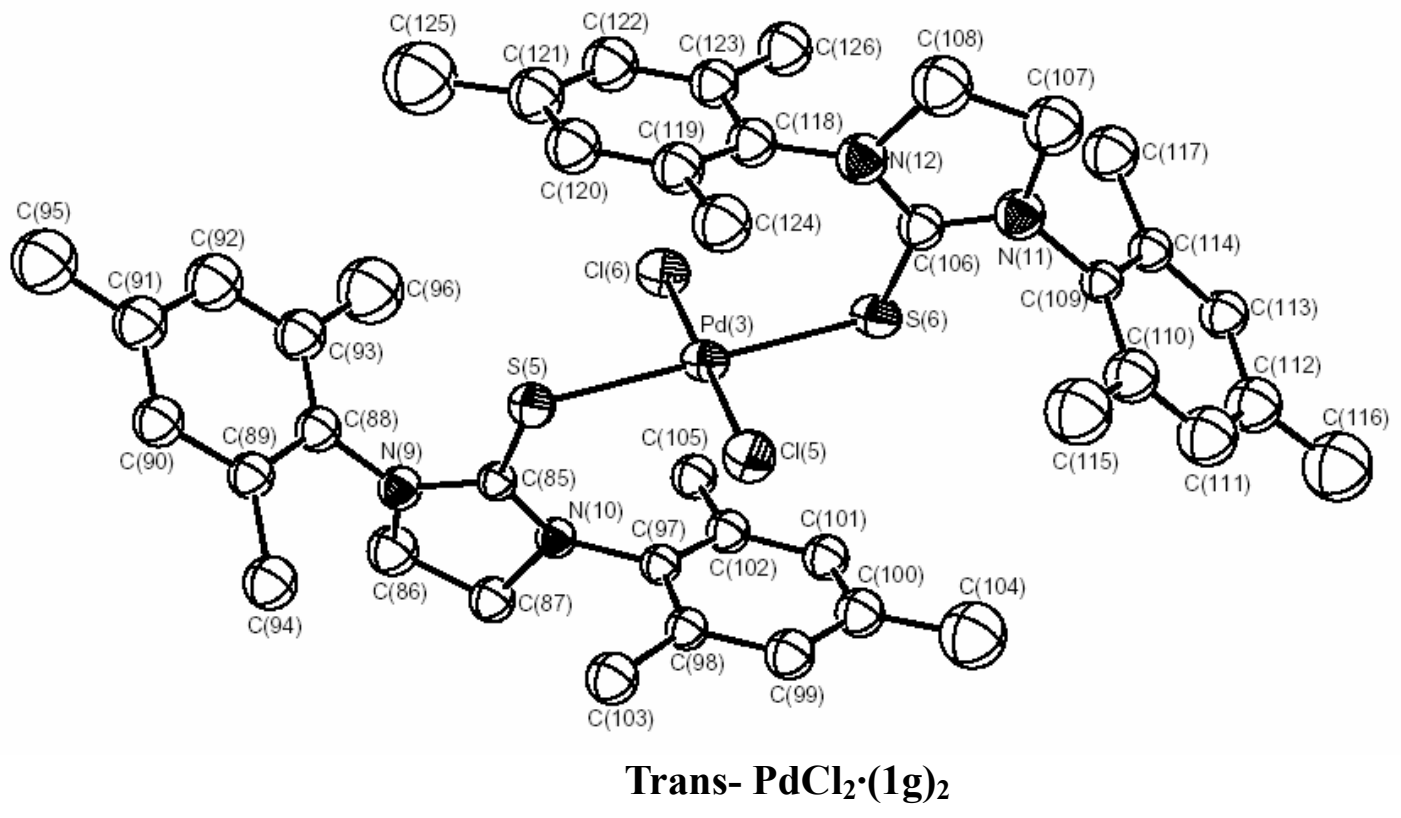


Table 1. Crystal data and structure refinement for $\mathrm{PdCl}_{2}-(\mathbf{1 g})_{2}$.

Identification code

Empirical formula

Formula weight

Temperature

Wavelength

Crystal system

Space group

Unit cell dimensions

Volume

Z

Density (calculated)

Absorption coefficient

$\mathrm{F}(000)$

Crystal size

Theta range for data collection

Index ranges

Reflections collected

Independent reflections

Completeness to theta $=25.57^{\circ}$

Absorption correction

Refinement method

Data / restraints / parameters

Goodness-of-fit on $\mathrm{F}^{2}$

Final $\mathrm{R}$ indices [I $>2 \operatorname{sigma}(\mathrm{I})]$

$\mathrm{R}$ indices (all data)

Largest diff. peak and hole
$\mathrm{PdCl}_{2}-(\mathbf{1 g})_{2}$.

$\mathrm{C}_{42.50} \mathrm{H}_{53.50} \mathrm{Cl}_{3} \mathrm{~N}_{4} \mathrm{O}_{0.25} \mathrm{Pd} \mathrm{S}_{2}$

901.26

253(2) K

$0.71073 \AA$

Monoclinic

P $2{ }_{1} / \mathrm{a}$

$\mathrm{a}=21.560(4) \AA$

$\alpha=90^{\circ}$.

$\mathrm{b}=31.285(6) \AA$

$\beta=97.14(3)^{\circ}$.

$\mathrm{c}=27.132(5) \AA$ $\gamma=90^{\circ}$.

18159(6) $\AA^{3}$

16

$1.319 \mathrm{Mg} / \mathrm{m}^{3}$

$0.711 \mathrm{~mm}^{-1}$

7480

$0.4 \times 0.2 \times 0.15 \mathrm{~mm}^{3}$

1.00 to $25.57^{\circ}$.

$-24<=\mathrm{h}<=24,-35<=\mathrm{k}<=35,-32<=1<=32$

60980

$23410[\mathrm{R}(\mathrm{int})=0.0672]$

$68.8 \%$

None

Full-matrix least-squares on $\mathrm{F}^{2}$

$23410 / 18$ / 956

0.879

$\mathrm{R} 1=0.0806, \mathrm{wR} 2=0.2247$

$\mathrm{R} 1=0.1884, \mathrm{wR} 2=0.2675$

2.155 and -1.090 e. $\AA^{-3}$ 
Table 3. Bond lengths $[\AA]$ and angles $\left[{ }^{\circ}\right]$ for $\operatorname{PdCl}_{2}-(\mathbf{1 g})_{2}$..

\begin{tabular}{|c|c|c|c|}
\hline $\operatorname{Pd}(1)-S(1)$ & $2.279(4)$ & $\mathrm{N}(4)-\mathrm{C}(34)$ & $1.437(15)$ \\
\hline $\operatorname{Pd}(1)-S(2)$ & $2.310(4)$ & $\mathrm{N}(4)-\mathrm{C}(24)$ & $1.461(13)$ \\
\hline $\operatorname{Pd}(1)-\mathrm{Cl}(2)$ & $2.337(4)$ & $\mathrm{N}(5)-\mathrm{C}(43)$ & $1.354(14)$ \\
\hline $\mathrm{Pd}(1)-\mathrm{Cl}(1)$ & $2.362(4)$ & $\mathrm{N}(5)-\mathrm{C}(46)$ & $1.442(15)$ \\
\hline $\operatorname{Pd}(2)-S(3)$ & $2.282(4)$ & $\mathrm{N}(5)-\mathrm{C}(44)$ & $1.484(14)$ \\
\hline $\operatorname{Pd}(2)-S(4)$ & $2.297(4)$ & $\mathrm{N}(6)-\mathrm{C}(43)$ & $1.324(14)$ \\
\hline $\operatorname{Pd}(2)-\mathrm{Cl}(3)$ & $2.346(5)$ & $N(6)-C(55)$ & $1.401(15)$ \\
\hline $\mathrm{Pd}(2)-\mathrm{Cl}(4)$ & $2.358(5)$ & $\mathrm{N}(6)-\mathrm{C}(45)$ & $1.472(13)$ \\
\hline $\operatorname{Pd}(3)-S(6)$ & $2.310(4)$ & $N(7)-C(64)$ & $1.309(14)$ \\
\hline $\operatorname{Pd}(3)-S(5)$ & $2.311(4)$ & $\mathrm{N}(7)-\mathrm{C}(67)$ & $1.409(15)$ \\
\hline $\operatorname{Pd}(3)-\mathrm{Cl}(5)$ & $2.318(4)$ & $N(7)-C(65)$ & $1.498(14)$ \\
\hline $\operatorname{Pd}(3)-\mathrm{Cl}(6)$ & $2.320(4)$ & $\mathrm{N}(8)-\mathrm{C}(64)$ & $1.385(14)$ \\
\hline $\operatorname{Pd}(4)-S(8)$ & $2.317(4)$ & $\mathrm{N}(8)-\mathrm{C}(76)$ & $1.440(16)$ \\
\hline $\operatorname{Pd}(4)-\mathrm{Cl}(8)$ & $2.323(4)$ & $\mathrm{N}(8)-\mathrm{C}(66)$ & $1.507(14)$ \\
\hline $\operatorname{Pd}(4)-S(7)$ & $2.325(4)$ & $\mathrm{N}(9)-\mathrm{C}(85)$ & $1.343(15)$ \\
\hline $\operatorname{Pd}(4)-\mathrm{Cl}(7)$ & $2.325(4)$ & $\mathrm{N}(9)-\mathrm{C}(88)$ & $1.401(14)$ \\
\hline$S(1)-C(1)$ & $1.662(10)$ & $\mathrm{N}(9)-\mathrm{C}(86)$ & $1.454(15)$ \\
\hline$S(2)-C(22)$ & $1.703(10)$ & $\mathrm{N}(10)-\mathrm{C}(85)$ & $1.359(12)$ \\
\hline$S(3)-C(43)$ & $1.708(10)$ & $\mathrm{N}(10)-\mathrm{C}(97)$ & $1.446(15)$ \\
\hline$S(4)-C(64)$ & $1.693(10)$ & $\mathrm{N}(10)-\mathrm{C}(87)$ & $1.483(15)$ \\
\hline$S(5)-C(85)$ & $1.676(12)$ & $\mathrm{N}(11)-\mathrm{C}(106)$ & $1.266(16)$ \\
\hline$S(6)-C(106)$ & $1.726(13)$ & $\mathrm{N}(11)-\mathrm{C}(109)$ & $1.461(14)$ \\
\hline$S(7)-C(127)$ & $1.685(12)$ & $\mathrm{N}(11)-\mathrm{C}(107)$ & $1.520(17)$ \\
\hline$S(8)-C(148)$ & $1.662(13)$ & $\mathrm{N}(12)-\mathrm{C}(106)$ & $1.376(14)$ \\
\hline $\mathrm{N}(1)-\mathrm{C}(1)$ & $1.343(13)$ & $\mathrm{N}(12)-\mathrm{C}(118)$ & $1.401(17)$ \\
\hline $\mathrm{N}(1)-\mathrm{C}(4)$ & $1.407(14)$ & $\mathrm{N}(12)-\mathrm{C}(108)$ & $1.490(17)$ \\
\hline $\mathrm{N}(1)-\mathrm{C}(2)$ & $1.512(13)$ & $\mathrm{N}(13)-\mathrm{C}(127)$ & $1.354(15)$ \\
\hline $\mathrm{N}(2)-\mathrm{C}(1)$ & $1.353(13)$ & $\mathrm{N}(13)-\mathrm{C}(130)$ & $1.490(15)$ \\
\hline $\mathrm{N}(2)-\mathrm{C}(13)$ & $1.448(15)$ & $\mathrm{N}(13)-\mathrm{C}(128)$ & $1.536(15)$ \\
\hline $\mathrm{N}(2)-\mathrm{C}(3)$ & $1.459(13)$ & $\mathrm{N}(14)-\mathrm{C}(127)$ & $1.303(12)$ \\
\hline $\mathrm{N}(3)-\mathrm{C}(22)$ & $1.368(14)$ & $\mathrm{N}(14)-\mathrm{C}(139)$ & $1.446(15)$ \\
\hline $\mathrm{N}(3)-\mathrm{C}(23)$ & $1.469(13)$ & $\mathrm{N}(14)-\mathrm{C}(129)$ & $1.500(15)$ \\
\hline $\mathrm{N}(3)-\mathrm{C}(25)$ & $1.500(16)$ & $\mathrm{N}(15)-\mathrm{C}(151)$ & $1.373(16)$ \\
\hline $\mathrm{N}(4)-\mathrm{C}(22)$ & $1.356(14)$ & $\mathrm{N}(15)-\mathrm{C}(148)$ & $1.407(14)$ \\
\hline
\end{tabular}




\begin{tabular}{|c|c|c|c|}
\hline $\mathrm{N}(15)-\mathrm{C}(149)$ & $1.436(18)$ & $C(35)-C(40)$ & $1.55(2)$ \\
\hline $\mathrm{N}(16)-\mathrm{C}(148)$ & $1.302(17)$ & $C(36)-C(37)$ & $1.353(19)$ \\
\hline $\mathrm{N}(16)-\mathrm{C}(160)$ & $1.447(15)$ & $\mathrm{C}(37)-\mathrm{C}(38)$ & $1.369(19)$ \\
\hline $\mathrm{N}(16)-\mathrm{C}(150)$ & $1.472(18)$ & $\mathrm{C}(37)-\mathrm{C}(41)$ & $1.49(2)$ \\
\hline$C(2)-C(3)$ & $1.531(17)$ & $\mathrm{C}(38)-\mathrm{C}(39)$ & $1.318(18)$ \\
\hline$C(4)-C(9)$ & $1.365(17)$ & $C(39)-C(42)$ & $1.509(18)$ \\
\hline$C(4)-C(5)$ & $1.370(17)$ & $\mathrm{C}(44)-\mathrm{C}(45)$ & $1.545(18)$ \\
\hline$C(5)-C(6)$ & $1.394(18)$ & $C(46)-C(51)$ & $1.373(18)$ \\
\hline$C(5)-C(10)$ & $1.491(18)$ & $\mathrm{C}(46)-\mathrm{C}(47)$ & $1.434(19)$ \\
\hline$C(6)-C(7)$ & $1.397(19)$ & $C(47)-C(48)$ & $1.354(18)$ \\
\hline$C(7)-C(8)$ & $1.414(18)$ & $C(47)-C(52)$ & $1.474(19)$ \\
\hline$C(7)-C(11)$ & $1.529(19)$ & $C(48)-C(49)$ & $1.358(19)$ \\
\hline$C(8)-C(9)$ & $1.344(17)$ & $C(49)-C(50)$ & $1.282(19)$ \\
\hline$C(9)-C(12)$ & $1.492(18)$ & $C(49)-C(53)$ & $1.54(2)$ \\
\hline$C(13)-C(18)$ & $1.349(18)$ & $C(50)-C(51)$ & $1.297(17)$ \\
\hline$C(13)-C(14)$ & $1.397(18)$ & $\mathrm{C}(51)-\mathrm{C}(54)$ & $1.48(2)$ \\
\hline$C(14)-C(15)$ & $1.423(16)$ & $C(55)-C(56)$ & $1.401(18)$ \\
\hline$C(14)-C(19)$ & $1.511(18)$ & $C(55)-C(60)$ & $1.405(18)$ \\
\hline$C(15)-C(16)$ & $1.451(18)$ & $C(56)-C(57)$ & $1.434(19)$ \\
\hline$C(16)-C(17)$ & $1.365(19)$ & $C(56)-C(61)$ & $1.48(2)$ \\
\hline$C(16)-C(20)$ & $1.59(2)$ & $C(57)-C(58)$ & $1.35(2)$ \\
\hline$C(17)-C(18)$ & $1.426(17)$ & $\mathrm{C}(58)-\mathrm{C}(59)$ & $1.391(19)$ \\
\hline$C(18)-C(21)$ & $1.53(2)$ & $\mathrm{C}(58)-\mathrm{C}(62)$ & $1.47(2)$ \\
\hline$C(23)-C(24)$ & $1.504(18)$ & $\mathrm{C}(59)-\mathrm{C}(60)$ & $1.415(17)$ \\
\hline$C(25)-C(30)$ & $1.358(18)$ & $\mathrm{C}(60)-\mathrm{C}(63)$ & $1.508(19)$ \\
\hline$C(25)-C(26)$ & $1.405(18)$ & $C(65)-C(66)$ & $1.484(18)$ \\
\hline$C(26)-C(27)$ & $1.398(17)$ & $C(67)-C(68)$ & $1.373(18)$ \\
\hline$C(26)-C(31)$ & $1.524(18)$ & $C(67)-C(72)$ & $1.442(17)$ \\
\hline$C(27)-C(28)$ & $1.384(18)$ & $\mathrm{C}(68)-\mathrm{C}(69)$ & $1.398(19)$ \\
\hline$C(28)-C(29)$ & $1.345(18)$ & $\mathrm{C}(68)-\mathrm{C}(73)$ & $1.536(19)$ \\
\hline$C(28)-C(32)$ & $1.445(19)$ & $\mathrm{C}(69)-\mathrm{C}(70)$ & $1.390(19)$ \\
\hline $\mathrm{C}(29)-\mathrm{C}(30)$ & $1.373(17)$ & $\mathrm{C}(70)-\mathrm{C}(71)$ & $1.351(19)$ \\
\hline $\mathrm{C}(30)-\mathrm{C}(33)$ & $1.490(19)$ & $C(70)-C(74)$ & $1.51(2)$ \\
\hline $\mathrm{C}(34)-\mathrm{C}(39)$ & $1.390(17)$ & $\mathrm{C}(71)-\mathrm{C}(72)$ & $1.432(18)$ \\
\hline$C(34)-C(35)$ & $1.403(18)$ & $C(72)-C(75)$ & $1.472(18)$ \\
\hline$C(35)-C(36)$ & $1.406(19)$ & $\mathrm{C}(76)-\mathrm{C}(81)$ & $1.384(17)$ \\
\hline
\end{tabular}




\begin{tabular}{|c|c|c|c|}
\hline$C(76)-C(77)$ & $1.434(19)$ & $\mathrm{C}(114)-\mathrm{C}(117)$ & $1.538(18)$ \\
\hline $\mathrm{C}(77)-\mathrm{C}(78)$ & $1.408(18)$ & $\mathrm{C}(118)-\mathrm{C}(123)$ & $1.410(18)$ \\
\hline $\mathrm{C}(77)-\mathrm{C}(82)$ & $1.499(18)$ & $\mathrm{C}(118)-\mathrm{C}(119)$ & $1.428(15)$ \\
\hline $\mathrm{C}(78)-\mathrm{C}(79)$ & $1.367(18)$ & $C(119)-C(120)$ & $1.332(19)$ \\
\hline $\mathrm{C}(79)-\mathrm{C}(80)$ & $1.344(19)$ & $\mathrm{C}(119)-\mathrm{C}(124)$ & $1.528(18)$ \\
\hline $\mathrm{C}(79)-\mathrm{C}(83)$ & $1.54(2)$ & $C(120)-C(121)$ & $1.35(2)$ \\
\hline $\mathrm{C}(80)-\mathrm{C}(81)$ & $1.385(16)$ & $C(121)-C(122)$ & $1.424(17)$ \\
\hline $\mathrm{C}(81)-\mathrm{C}(84)$ & $1.495(18)$ & $C(121)-C(125)$ & $1.50(2)$ \\
\hline $\mathrm{C}(86)-\mathrm{C}(87)$ & $1.505(17)$ & $\mathrm{C}(122)-\mathrm{C}(123)$ & $1.360(19)$ \\
\hline C(88)-C(89) & $1.351(14)$ & $C(123)-C(126)$ & $1.511(16)$ \\
\hline $\mathrm{C}(88)-\mathrm{C}(93)$ & $1.357(17)$ & $\mathrm{C}(128)-\mathrm{C}(129)$ & $1.515(16)$ \\
\hline C(89)-C(90) & $1.418(16)$ & $\mathrm{C}(130)-\mathrm{C}(131)$ & $1.304(14)$ \\
\hline C(89)-C(94) & $1.492(16)$ & $C(130)-C(135)$ & $1.422(18)$ \\
\hline $\mathrm{C}(90)-\mathrm{C}(91)$ & $1.371(19)$ & $C(131)-C(132)$ & $1.411(17)$ \\
\hline $\mathrm{C}(91)-\mathrm{C}(92)$ & $1.374(16)$ & $C(131)-C(136)$ & $1.557(16)$ \\
\hline $\mathrm{C}(91)-\mathrm{C}(95)$ & $1.469(19)$ & $\mathrm{C}(132)-\mathrm{C}(133)$ & $1.359(18)$ \\
\hline $\mathrm{C}(92)-\mathrm{C}(93)$ & $1.389(17)$ & $C(133)-C(134)$ & $1.406(16)$ \\
\hline $\mathrm{C}(93)-\mathrm{C}(96)$ & $1.457(17)$ & $\mathrm{C}(133)-\mathrm{C}(137)$ & $1.493(19)$ \\
\hline C(97)-C(98) & $1.371(16)$ & $\mathrm{C}(134)-\mathrm{C}(135)$ & $1.412(17)$ \\
\hline $\mathrm{C}(97)-\mathrm{C}(102)$ & $1.415(14)$ & $\mathrm{C}(135)-\mathrm{C}(138)$ & $1.552(16)$ \\
\hline C(98)-C(99) & $1.385(17)$ & $C(139)-C(140)$ & $1.393(16)$ \\
\hline $\mathrm{C}(98)-\mathrm{C}(103)$ & $1.484(15)$ & $\mathrm{C}(139)-\mathrm{C}(144)$ & $1.396(14)$ \\
\hline C(99)-C(100) & $1.424(15)$ & $\mathrm{C}(140)-\mathrm{C}(141)$ & $1.360(17)$ \\
\hline $\mathrm{C}(100)-\mathrm{C}(101)$ & $1.338(18)$ & $C(140)-C(145)$ & $1.514(15)$ \\
\hline$C(100)-C(104)$ & $1.52(2)$ & $\mathrm{C}(141)-\mathrm{C}(142)$ & $1.418(15)$ \\
\hline $\mathrm{C}(101)-\mathrm{C}(102)$ & $1.377(18)$ & $\mathrm{C}(142)-\mathrm{C}(143)$ & $1.338(18)$ \\
\hline$C(102)-C(105)$ & $1.499(16)$ & $\mathrm{C}(142)-\mathrm{C}(146)$ & $1.50(2)$ \\
\hline$C(107)-C(108)$ & $1.496(18)$ & $C(143)-C(144)$ & $1.389(18)$ \\
\hline$C(109)-C(114)$ & $1.378(14)$ & $C(144)-C(147)$ & $1.472(17)$ \\
\hline $\mathrm{C}(109)-\mathrm{C}(110)$ & $1.393(18)$ & $\mathrm{C}(149)-\mathrm{C}(150)$ & $1.57(2)$ \\
\hline$C(110)-C(111)$ & $1.364(18)$ & $C(151)-C(156)$ & $1.362(18)$ \\
\hline$C(110)-C(115)$ & $1.533(17)$ & $\mathrm{C}(151)-\mathrm{C}(152)$ & $1.429(15)$ \\
\hline$C(111)-C(112)$ & $1.330(17)$ & $C(152)-C(153)$ & $1.37(2)$ \\
\hline$C(112)-C(113)$ & $1.377(19)$ & $C(152)-C(157)$ & $1.495(19)$ \\
\hline$C(112)-C(116)$ & $1.48(2)$ & $\mathrm{C}(153)-\mathrm{C}(154)$ & $1.36(2)$ \\
\hline$C(113)-C(114)$ & $1.385(16)$ & $\mathrm{C}(154)-\mathrm{C}(155)$ & $1.415(17)$ \\
\hline
\end{tabular}




\begin{tabular}{|c|c|c|c|}
\hline$C(154)-C(158)$ & $1.50(2)$ & $\mathrm{S}(3)-\mathrm{Pd}(2)-\mathrm{Cl}(4)$ & $88.41(18)$ \\
\hline$C(155)-C(156)$ & $1.338(19)$ & $\mathrm{S}(4)-\mathrm{Pd}(2)-\mathrm{Cl}(4)$ & $176.90(14)$ \\
\hline$C(156)-C(159)$ & $1.548(16)$ & $\mathrm{Cl}(3)-\operatorname{Pd}(2)-\mathrm{Cl}(4)$ & $93.2(3)$ \\
\hline$C(160)-C(165)$ & $1.356(14)$ & $S(6)-P d(3)-S(5)$ & $179.80(17)$ \\
\hline$C(160)-C(161)$ & $1.427(18)$ & $\mathrm{S}(6)-\mathrm{Pd}(3)-\mathrm{Cl}(5)$ & $89.60(15)$ \\
\hline$C(161)-C(162)$ & $1.376(18)$ & $\mathrm{S}(5)-\mathrm{Pd}(3)-\mathrm{Cl}(5)$ & $90.42(14)$ \\
\hline$C(161)-C(166)$ & $1.548(18)$ & $\mathrm{S}(6)-\mathrm{Pd}(3)-\mathrm{Cl}(6)$ & $90.56(15)$ \\
\hline$C(162)-C(163)$ & $1.389(17)$ & $\mathrm{S}(5)-\mathrm{Pd}(3)-\mathrm{Cl}(6)$ & $89.42(15)$ \\
\hline$C(163)-C(164)$ & $1.396(18)$ & $\mathrm{Cl}(5)-\mathrm{Pd}(3)-\mathrm{Cl}(6)$ & $179.63(19)$ \\
\hline$C(163)-C(167)$ & $1.49(2)$ & $\mathrm{S}(8)-\mathrm{Pd}(4)-\mathrm{Cl}(8)$ & $89.19(15)$ \\
\hline$C(164)-C(165)$ & $1.382(17)$ & $\mathrm{S}(8)-\mathrm{Pd}(4)-\mathrm{S}(7)$ & $179.6(2)$ \\
\hline$C(165)-C(168)$ & $1.502(18)$ & $\mathrm{Cl}(8)-\mathrm{Pd}(4)-\mathrm{S}(7)$ & $91.18(15)$ \\
\hline $\mathrm{Cl}(9)-\mathrm{C}(169)$ & $1.75(2)$ & $\mathrm{S}(8)-\mathrm{Pd}(4)-\mathrm{Cl}(7)$ & $90.82(14)$ \\
\hline $\mathrm{Cl}(10)-\mathrm{O}(1) \# 1$ & $1.50(3)$ & $\mathrm{Cl}(8)-\mathrm{Pd}(4)-\mathrm{Cl}(7)$ & $179.59(17)$ \\
\hline $\mathrm{Cl}(10)-\mathrm{C}(169)$ & $1.78(2)$ & $\mathrm{S}(7)-\mathrm{Pd}(4)-\mathrm{Cl}(7)$ & $88.81(15)$ \\
\hline $\mathrm{Cl}(11)-\mathrm{C}(171)$ & $1.21(4)$ & $C(1)-S(1)-P d(1)$ & $111.1(4)$ \\
\hline $\mathrm{Cl}(11)-\mathrm{Cl}(13)$ & $1.226(14)$ & $C(22)-S(2)-P d(1)$ & $110.3(5)$ \\
\hline $\mathrm{Cl}(11)-\mathrm{Cl}(14)$ & $1.71(2)$ & $\mathrm{C}(43)-\mathrm{S}(3)-\mathrm{Pd}(2)$ & $111.1(4)$ \\
\hline $\mathrm{Cl}(11)-\mathrm{C}(170)$ & $1.78(2)$ & $\mathrm{C}(64)-\mathrm{S}(4)-\mathrm{Pd}(2)$ & $111.0(4)$ \\
\hline $\mathrm{Cl}(12)-\mathrm{Cl}(14)$ & $1.201(15)$ & $\mathrm{C}(85)-\mathrm{S}(5)-\mathrm{Pd}(3)$ & $110.6(4)$ \\
\hline $\mathrm{Cl}(12)-\mathrm{C}(170)$ & $1.79(2)$ & $\mathrm{C}(106)-\mathrm{S}(6)-\mathrm{Pd}(3)$ & $111.8(4)$ \\
\hline $\mathrm{Cl}(13)-\mathrm{C}(171)$ & $1.76(2)$ & $\mathrm{C}(127)-\mathrm{S}(7)-\mathrm{Pd}(4)$ & $110.6(4)$ \\
\hline $\mathrm{Cl}(14)-\mathrm{C}(170)$ & $1.27(4)$ & $\mathrm{C}(148)-\mathrm{S}(8)-\mathrm{Pd}(4)$ & $110.7(4)$ \\
\hline $\mathrm{Cl}(14)-\mathrm{C}(171)$ & $1.77(2)$ & $\mathrm{C}(1)-\mathrm{N}(1)-\mathrm{C}(4)$ & $126.4(9)$ \\
\hline$C(170)-C(171)$ & $1.63(6)$ & $\mathrm{C}(1)-\mathrm{N}(1)-\mathrm{C}(2)$ & $112.5(9)$ \\
\hline \multirow[t]{2}{*}{$\mathrm{O}(1)-\mathrm{Cl}(10) \# 2$} & $1.50(3)$ & $\mathrm{C}(4)-\mathrm{N}(1)-\mathrm{C}(2)$ & $121.0(9)$ \\
\hline & & $\mathrm{C}(1)-\mathrm{N}(2)-\mathrm{C}(13)$ & $126.1(9)$ \\
\hline $\mathrm{S}(1)-\mathrm{Pd}(1)-\mathrm{S}(2)$ & $90.8(2)$ & $\mathrm{C}(1)-\mathrm{N}(2)-\mathrm{C}(3)$ & $113.6(10)$ \\
\hline $\mathrm{S}(1)-\mathrm{Pd}(1)-\mathrm{Cl}(2)$ & $88.96(16)$ & $\mathrm{C}(13)-\mathrm{N}(2)-\mathrm{C}(3)$ & $118.5(9)$ \\
\hline $\mathrm{S}(2)-\mathrm{Pd}(1)-\mathrm{Cl}(2)$ & $178.09(13)$ & $\mathrm{C}(22)-\mathrm{N}(3)-\mathrm{C}(23)$ & $111.4(10)$ \\
\hline $\mathrm{S}(1)-\mathrm{Pd}(1)-\mathrm{Cl}(1)$ & $176.52(13)$ & $\mathrm{C}(22)-\mathrm{N}(3)-\mathrm{C}(25)$ & $126.4(9)$ \\
\hline $\mathrm{S}(2)-\mathrm{Pd}(1)-\mathrm{Cl}(1)$ & $88.14(16)$ & $\mathrm{C}(23)-\mathrm{N}(3)-\mathrm{C}(25)$ & $120.3(10)$ \\
\hline $\mathrm{Cl}(2)-\mathrm{Pd}(1)-\mathrm{Cl}(1)$ & $92.26(17)$ & $\mathrm{C}(22)-\mathrm{N}(4)-\mathrm{C}(34)$ & $125.5(9)$ \\
\hline$S(3)-P d(2)-S(4)$ & $90.9(2)$ & $\mathrm{C}(22)-\mathrm{N}(4)-\mathrm{C}(24)$ & 107.1(10) \\
\hline $\mathrm{S}(3)-\mathrm{Pd}(2)-\mathrm{Cl}(3)$ & $177.09(15)$ & $\mathrm{C}(34)-\mathrm{N}(4)-\mathrm{C}(24)$ & $127.3(10)$ \\
\hline $\mathrm{S}(4)-\mathrm{Pd}(2)-\mathrm{Cl}(3)$ & $87.61(18)$ & $\mathrm{C}(43)-\mathrm{N}(5)-\mathrm{C}(46)$ & $127.7(9)$ \\
\hline
\end{tabular}




\begin{tabular}{|c|c|c|c|}
\hline $\mathrm{C}(43)-\mathrm{N}(5)-\mathrm{C}(44)$ & $108.7(10)$ & $\mathrm{N}(1)-\mathrm{C}(1)-\mathrm{S}(1)$ & $120.2(8)$ \\
\hline $\mathrm{C}(46)-\mathrm{N}(5)-\mathrm{C}(44)$ & $120.8(10)$ & $\mathrm{N}(2)-\mathrm{C}(1)-\mathrm{S}(1)$ & 131.9(9) \\
\hline $\mathrm{C}(43)-\mathrm{N}(6)-\mathrm{C}(55)$ & $127.9(10)$ & $\mathrm{N}(1)-\mathrm{C}(2)-\mathrm{C}(3)$ & 101.2(9) \\
\hline $\mathrm{C}(43)-\mathrm{N}(6)-\mathrm{C}(45)$ & $112.2(10)$ & $\mathrm{N}(2)-\mathrm{C}(3)-\mathrm{C}(2)$ & 103.3(9) \\
\hline $\mathrm{C}(55)-\mathrm{N}(6)-\mathrm{C}(45)$ & $119.9(10)$ & $\mathrm{C}(9)-\mathrm{C}(4)-\mathrm{C}(5)$ & $122.7(12)$ \\
\hline $\mathrm{C}(64)-\mathrm{N}(7)-\mathrm{C}(67)$ & $126.4(10)$ & $\mathrm{C}(9)-\mathrm{C}(4)-\mathrm{N}(1)$ & $120.3(12)$ \\
\hline $\mathrm{C}(64)-\mathrm{N}(7)-\mathrm{C}(65)$ & $113.1(10)$ & $\mathrm{C}(5)-\mathrm{C}(4)-\mathrm{N}(1)$ & $116.9(12)$ \\
\hline $\mathrm{C}(67)-\mathrm{N}(7)-\mathrm{C}(65)$ & $120.3(10)$ & $C(4)-C(5)-C(6)$ & $118.7(13)$ \\
\hline $\mathrm{C}(64)-\mathrm{N}(8)-\mathrm{C}(76)$ & $126.8(9)$ & $\mathrm{C}(4)-\mathrm{C}(5)-\mathrm{C}(10)$ & $120.9(13)$ \\
\hline $\mathrm{C}(64)-\mathrm{N}(8)-\mathrm{C}(66)$ & $107.8(10)$ & $C(6)-C(5)-C(10)$ & $120.3(13)$ \\
\hline $\mathrm{C}(76)-\mathrm{N}(8)-\mathrm{C}(66)$ & $124.4(10)$ & $C(5)-C(6)-C(7)$ & $121.5(15)$ \\
\hline $\mathrm{C}(85)-\mathrm{N}(9)-\mathrm{C}(88)$ & $125.8(11)$ & $C(6)-C(7)-C(8)$ & $114.5(14)$ \\
\hline $\mathrm{C}(85)-\mathrm{N}(9)-\mathrm{C}(86)$ & $110.7(10)$ & $C(6)-C(7)-C(11)$ & $119.2(14)$ \\
\hline $\mathrm{C}(88)-\mathrm{N}(9)-\mathrm{C}(86)$ & $122.7(12)$ & $C(8)-C(7)-C(11)$ & $126.3(14)$ \\
\hline $\mathrm{C}(85)-\mathrm{N}(10)-\mathrm{C}(97)$ & $127.2(10)$ & $\mathrm{C}(9)-\mathrm{C}(8)-\mathrm{C}(7)$ & $125.5(15)$ \\
\hline $\mathrm{C}(85)-\mathrm{N}(10)-\mathrm{C}(87)$ & 110.1(9) & $\mathrm{C}(8)-\mathrm{C}(9)-\mathrm{C}(4)$ & $116.9(13)$ \\
\hline $\mathrm{C}(97)-\mathrm{N}(10)-\mathrm{C}(87)$ & 119.4(9) & $\mathrm{C}(8)-\mathrm{C}(9)-\mathrm{C}(12)$ & $119.7(14)$ \\
\hline $\mathrm{C}(106)-\mathrm{N}(11)-\mathrm{C}(109)$ & $125.8(12)$ & $\mathrm{C}(4)-\mathrm{C}(9)-\mathrm{C}(12)$ & $123.4(13)$ \\
\hline $\mathrm{C}(106)-\mathrm{N}(11)-\mathrm{C}(107)$ & $115.6(11)$ & $\mathrm{C}(18)-\mathrm{C}(13)-\mathrm{C}(14)$ & $125.1(13)$ \\
\hline C(109)-N(11)-C(107) & $118.2(11)$ & $\mathrm{C}(18)-\mathrm{C}(13)-\mathrm{N}(2)$ & $117.5(13)$ \\
\hline $\mathrm{C}(106)-\mathrm{N}(12)-\mathrm{C}(118)$ & $126.1(13)$ & $\mathrm{C}(14)-\mathrm{C}(13)-\mathrm{N}(2)$ & $117.3(12)$ \\
\hline $\mathrm{C}(106)-\mathrm{N}(12)-\mathrm{C}(108)$ & $105.7(11)$ & $C(13)-C(14)-C(15)$ & $115.3(12)$ \\
\hline $\mathrm{C}(118)-\mathrm{N}(12)-\mathrm{C}(108)$ & $126.4(12)$ & $C(13)-C(14)-C(19)$ & $124.3(12)$ \\
\hline $\mathrm{C}(127)-\mathrm{N}(13)-\mathrm{C}(130)$ & $127.3(10)$ & $C(15)-C(14)-C(19)$ & $120.4(12)$ \\
\hline $\mathrm{C}(127)-\mathrm{N}(13)-\mathrm{C}(128)$ & 113.1(9) & $\mathrm{C}(14)-\mathrm{C}(15)-\mathrm{C}(16)$ & $121.4(13)$ \\
\hline $\mathrm{C}(130)-\mathrm{N}(13)-\mathrm{C}(128)$ & $119.2(10)$ & $C(17)-C(16)-C(15)$ & $118.3(13)$ \\
\hline $\mathrm{C}(127)-\mathrm{N}(14)-\mathrm{C}(139)$ & $128.5(11)$ & $C(17)-C(16)-C(20)$ & $122.5(14)$ \\
\hline $\mathrm{C}(127)-\mathrm{N}(14)-\mathrm{C}(129)$ & $110.6(10)$ & $C(15)-C(16)-C(20)$ & $119.2(13)$ \\
\hline C(139)-N(14)-C(129) & $118.7(9)$ & $C(16)-C(17)-C(18)$ & $121.0(14)$ \\
\hline $\mathrm{C}(151)-\mathrm{N}(15)-\mathrm{C}(148)$ & $125.8(12)$ & $\mathrm{C}(13)-\mathrm{C}(18)-\mathrm{C}(17)$ & $118.7(14)$ \\
\hline $\mathrm{C}(151)-\mathrm{N}(15)-\mathrm{C}(149)$ & $125.0(12)$ & $\mathrm{C}(13)-\mathrm{C}(18)-\mathrm{C}(21)$ & $124.9(13)$ \\
\hline $\mathrm{C}(148)-\mathrm{N}(15)-\mathrm{C}(149)$ & $106.4(11)$ & $\mathrm{C}(17)-\mathrm{C}(18)-\mathrm{C}(21)$ & $116.4(13)$ \\
\hline $\mathrm{C}(148)-\mathrm{N}(16)-\mathrm{C}(160)$ & $131.0(12)$ & $\mathrm{N}(4)-\mathrm{C}(22)-\mathrm{N}(3)$ & $111.3(9)$ \\
\hline $\mathrm{C}(148)-\mathrm{N}(16)-\mathrm{C}(150)$ & $116.1(12)$ & $\mathrm{N}(4)-\mathrm{C}(22)-\mathrm{S}(2)$ & 119.2(9) \\
\hline $\mathrm{C}(160)-\mathrm{N}(16)-\mathrm{C}(150)$ & $112.7(12)$ & $\mathrm{N}(3)-\mathrm{C}(22)-\mathrm{S}(2)$ & $129.5(9)$ \\
\hline $\mathrm{N}(1)-\mathrm{C}(1)-\mathrm{N}(2)$ & $107.9(9)$ & $\mathrm{N}(3)-\mathrm{C}(23)-\mathrm{C}(24)$ & $100.7(10)$ \\
\hline
\end{tabular}




\begin{tabular}{|c|c|c|c|}
\hline $\mathrm{N}(4)-\mathrm{C}(24)-\mathrm{C}(23)$ & $108.0(10)$ & $\mathrm{C}(47)-\mathrm{C}(46)-\mathrm{N}(5)$ & $117.4(13)$ \\
\hline$C(30)-C(25)-C(26)$ & $127.5(13)$ & $\mathrm{C}(48)-\mathrm{C}(47)-\mathrm{C}(46)$ & $114.9(14)$ \\
\hline $\mathrm{C}(30)-\mathrm{C}(25)-\mathrm{N}(3)$ & $116.2(12)$ & $C(48)-C(47)-C(52)$ & $121.7(15)$ \\
\hline $\mathrm{C}(26)-\mathrm{C}(25)-\mathrm{N}(3)$ & $116.2(12)$ & $\mathrm{C}(46)-\mathrm{C}(47)-\mathrm{C}(52)$ & $123.4(13)$ \\
\hline$C(27)-C(26)-C(25)$ & $114.6(13)$ & $\mathrm{C}(47)-\mathrm{C}(48)-\mathrm{C}(49)$ & $121.9(16)$ \\
\hline$C(27)-C(26)-C(31)$ & $121.2(13)$ & $\mathrm{C}(50)-\mathrm{C}(49)-\mathrm{C}(48)$ & $121.5(16)$ \\
\hline$C(25)-C(26)-C(31)$ & $124.3(12)$ & $C(50)-C(49)-C(53)$ & $119.6(16)$ \\
\hline$C(28)-C(27)-C(26)$ & $119.7(14)$ & $C(48)-C(49)-C(53)$ & $118.9(15)$ \\
\hline$C(29)-C(28)-C(27)$ & $120.6(14)$ & $C(49)-C(50)-C(51)$ & $121.4(17)$ \\
\hline $\mathrm{C}(29)-\mathrm{C}(28)-\mathrm{C}(32)$ & 121.1(14) & $C(50)-C(51)-C(46)$ & $121.2(15)$ \\
\hline$C(27)-C(28)-C(32)$ & $118.2(14)$ & $C(50)-C(51)-C(54)$ & $122.8(15)$ \\
\hline $\mathrm{C}(28)-\mathrm{C}(29)-\mathrm{C}(30)$ & $124.2(14)$ & $C(46)-C(51)-C(54)$ & $115.9(13)$ \\
\hline$C(25)-C(30)-C(29)$ & $113.3(13)$ & $\mathrm{N}(6)-\mathrm{C}(55)-\mathrm{C}(56)$ & $119.1(13)$ \\
\hline$C(25)-C(30)-C(33)$ & $126.5(13)$ & $\mathrm{N}(6)-\mathrm{C}(55)-\mathrm{C}(60)$ & $118.9(13)$ \\
\hline$C(29)-C(30)-C(33)$ & $120.2(13)$ & $C(56)-C(55)-C(60)$ & $121.9(13)$ \\
\hline$C(39)-C(34)-C(35)$ & 119.6(13) & $C(55)-C(56)-C(57)$ & $116.5(15)$ \\
\hline $\mathrm{C}(39)-\mathrm{C}(34)-\mathrm{N}(4)$ & $123.4(13)$ & $C(55)-C(56)-C(61)$ & $121.7(14)$ \\
\hline $\mathrm{C}(35)-\mathrm{C}(34)-\mathrm{N}(4)$ & $116.7(13)$ & $C(57)-C(56)-C(61)$ & $121.6(15)$ \\
\hline$C(34)-C(35)-C(36)$ & $118.1(15)$ & $C(58)-C(57)-C(56)$ & $123.9(17)$ \\
\hline$C(34)-C(35)-C(40)$ & $119.3(14)$ & $C(57)-C(58)-C(59)$ & $117.3(15)$ \\
\hline$C(36)-C(35)-C(40)$ & $122.6(15)$ & $\mathrm{C}(57)-\mathrm{C}(58)-\mathrm{C}(62)$ & $123.4(16)$ \\
\hline$C(37)-C(36)-C(35)$ & $118.3(16)$ & $\mathrm{C}(59)-\mathrm{C}(58)-\mathrm{C}(62)$ & $119.1(15)$ \\
\hline$C(36)-C(37)-C(38)$ & $123.3(16)$ & $C(58)-C(59)-C(60)$ & $123.3(14)$ \\
\hline$C(36)-C(37)-C(41)$ & $113.3(15)$ & $C(55)-C(60)-C(59)$ & $116.9(13)$ \\
\hline $\mathrm{C}(38)-\mathrm{C}(37)-\mathrm{C}(41)$ & $123.4(16)$ & $C(55)-C(60)-C(63)$ & $118.6(13)$ \\
\hline $\mathrm{C}(39)-\mathrm{C}(38)-\mathrm{C}(37)$ & $119.2(16)$ & $C(59)-C(60)-C(63)$ & $124.4(14)$ \\
\hline$C(38)-C(39)-C(34)$ & $121.4(14)$ & N(7)-C(64)-N(8) & $110.3(9)$ \\
\hline $\mathrm{C}(38)-\mathrm{C}(39)-\mathrm{C}(42)$ & $120.0(14)$ & N(7)-C(64)-S(4) & $120.1(9)$ \\
\hline$C(34)-C(39)-C(42)$ & $118.5(13)$ & $\mathrm{N}(8)-\mathrm{C}(64)-\mathrm{S}(4)$ & $129.0(9)$ \\
\hline $\mathrm{N}(6)-\mathrm{C}(43)-\mathrm{N}(5)$ & $112.1(9)$ & $\mathrm{C}(66)-\mathrm{C}(65)-\mathrm{N}(7)$ & $101.9(11)$ \\
\hline $\mathrm{N}(6)-\mathrm{C}(43)-\mathrm{S}(3)$ & 119.7(9) & $\mathrm{C}(65)-\mathrm{C}(66)-\mathrm{N}(8)$ & $105.3(10)$ \\
\hline $\mathrm{N}(5)-\mathrm{C}(43)-\mathrm{S}(3)$ & $128.1(9)$ & $\mathrm{C}(68)-\mathrm{C}(67)-\mathrm{N}(7)$ & $121.3(13)$ \\
\hline $\mathrm{N}(5)-\mathrm{C}(44)-\mathrm{C}(45)$ & $104.4(10)$ & $\mathrm{C}(68)-\mathrm{C}(67)-\mathrm{C}(72)$ & $121.3(13)$ \\
\hline $\mathrm{N}(6)-\mathrm{C}(45)-\mathrm{C}(44)$ & $101.4(10)$ & $\mathrm{N}(7)-\mathrm{C}(67)-\mathrm{C}(72)$ & $117.4(12)$ \\
\hline $\mathrm{C}(51)-\mathrm{C}(46)-\mathrm{C}(47)$ & $118.9(13)$ & $\mathrm{C}(67)-\mathrm{C}(68)-\mathrm{C}(69)$ & $120.3(14)$ \\
\hline $\mathrm{C}(51)-\mathrm{C}(46)-\mathrm{N}(5)$ & $123.5(13)$ & $\mathrm{C}(67)-\mathrm{C}(68)-\mathrm{C}(73)$ & $120.3(13)$ \\
\hline
\end{tabular}




\begin{tabular}{|c|c|c|c|}
\hline$C(69)-C(68)-C(73)$ & $119.4(14)$ & $\mathrm{C}(90)-\mathrm{C}(91)-\mathrm{C}(95)$ & $123.4(13)$ \\
\hline$C(70)-C(69)-C(68)$ & $120.7(15)$ & $\mathrm{C}(92)-\mathrm{C}(91)-\mathrm{C}(95)$ & $119.0(15)$ \\
\hline$C(71)-C(70)-C(69)$ & $118.8(15)$ & $\mathrm{C}(91)-\mathrm{C}(92)-\mathrm{C}(93)$ & $123.0(15)$ \\
\hline $\mathrm{C}(71)-\mathrm{C}(70)-\mathrm{C}(74)$ & $121.1(16)$ & $\mathrm{C}(88)-\mathrm{C}(93)-\mathrm{C}(92)$ & $117.9(13)$ \\
\hline$C(69)-C(70)-C(74)$ & $120.1(15)$ & $\mathrm{C}(88)-\mathrm{C}(93)-\mathrm{C}(96)$ & $120.9(14)$ \\
\hline $\mathrm{C}(70)-\mathrm{C}(71)-\mathrm{C}(72)$ & $124.3(15)$ & $\mathrm{C}(92)-\mathrm{C}(93)-\mathrm{C}(96)$ & $121.2(15)$ \\
\hline$C(71)-C(72)-C(67)$ & $114.6(12)$ & $\mathrm{C}(98)-\mathrm{C}(97)-\mathrm{C}(102)$ & $123.6(12)$ \\
\hline$C(71)-C(72)-C(75)$ & $123.6(13)$ & $\mathrm{C}(98)-\mathrm{C}(97)-\mathrm{N}(10)$ & $117.5(10)$ \\
\hline$C(67)-C(72)-C(75)$ & $121.8(13)$ & $\mathrm{C}(102)-\mathrm{C}(97)-\mathrm{N}(10)$ & $118.6(11)$ \\
\hline$C(81)-C(76)-C(77)$ & $120.5(13)$ & $\mathrm{C}(97)-\mathrm{C}(98)-\mathrm{C}(99)$ & $116.2(11)$ \\
\hline $\mathrm{C}(81)-\mathrm{C}(76)-\mathrm{N}(8)$ & $121.5(13)$ & $\mathrm{C}(97)-\mathrm{C}(98)-\mathrm{C}(103)$ & $125.0(12)$ \\
\hline $\mathrm{C}(77)-\mathrm{C}(76)-\mathrm{N}(8)$ & $117.5(13)$ & $\mathrm{C}(99)-\mathrm{C}(98)-\mathrm{C}(103)$ & $118.7(12)$ \\
\hline$C(78)-C(77)-C(76)$ & $116.8(13)$ & $\mathrm{C}(98)-\mathrm{C}(99)-\mathrm{C}(100)$ & $121.1(14)$ \\
\hline$C(78)-C(77)-C(82)$ & $123.2(14)$ & $C(101)-C(100)-C(99)$ & $119.8(15)$ \\
\hline$C(76)-C(77)-C(82)$ & $120.0(13)$ & $C(101)-C(100)-C(104)$ & $119.4(13)$ \\
\hline$C(79)-C(78)-C(77)$ & $122.0(14)$ & $C(99)-C(100)-C(104)$ & $120.7(14)$ \\
\hline $\mathrm{C}(80)-\mathrm{C}(79)-\mathrm{C}(78)$ & $118.7(14)$ & $C(100)-C(101)-C(102)$ & $121.5(13)$ \\
\hline$C(80)-C(79)-C(83)$ & $118.5(14)$ & $C(101)-C(102)-C(97)$ & $117.3(12)$ \\
\hline $\mathrm{C}(78)-\mathrm{C}(79)-\mathrm{C}(83)$ & $122.4(15)$ & $C(101)-C(102)-C(105)$ & $120.7(10)$ \\
\hline$C(79)-C(80)-C(81)$ & $123.9(14)$ & $C(97)-C(102)-C(105)$ & $121.9(12)$ \\
\hline $\mathrm{C}(76)-\mathrm{C}(81)-\mathrm{C}(80)$ & $117.9(13)$ & $\mathrm{N}(11)-\mathrm{C}(106)-\mathrm{N}(12)$ & $111.7(12)$ \\
\hline$C(76)-C(81)-C(84)$ & $117.2(12)$ & $\mathrm{N}(11)-\mathrm{C}(106)-\mathrm{S}(6)$ & $120.4(10)$ \\
\hline $\mathrm{C}(80)-\mathrm{C}(81)-\mathrm{C}(84)$ & $124.9(13)$ & $\mathrm{N}(12)-\mathrm{C}(106)-\mathrm{S}(6)$ & $128.0(11)$ \\
\hline $\mathrm{N}(9)-\mathrm{C}(85)-\mathrm{N}(10)$ & $109.8(10)$ & $\mathrm{C}(108)-\mathrm{C}(107)-\mathrm{N}(11)$ & $97.4(12)$ \\
\hline $\mathrm{N}(9)-\mathrm{C}(85)-\mathrm{S}(5)$ & $120.8(8)$ & $\mathrm{N}(12)-\mathrm{C}(108)-\mathrm{C}(107)$ & $109.2(12)$ \\
\hline $\mathrm{N}(10)-\mathrm{C}(85)-\mathrm{S}(5)$ & $129.4(9)$ & $C(114)-C(109)-C(110)$ & $120.5(12)$ \\
\hline $\mathrm{N}(9)-\mathrm{C}(86)-\mathrm{C}(87)$ & $104.5(11)$ & $\mathrm{C}(114)-\mathrm{C}(109)-\mathrm{N}(11)$ & $119.3(11)$ \\
\hline $\mathrm{N}(10)-\mathrm{C}(87)-\mathrm{C}(86)$ & $101.9(10)$ & $\mathrm{C}(110)-\mathrm{C}(109)-\mathrm{N}(11)$ & $120.1(10)$ \\
\hline C(89)-C(88)-C(93) & $121.7(13)$ & $C(111)-C(110)-C(109)$ & 119.1(13) \\
\hline $\mathrm{C}(89)-\mathrm{C}(88)-\mathrm{N}(9)$ & $116.5(12)$ & $C(111)-C(110)-C(115)$ & $120.8(15)$ \\
\hline $\mathrm{C}(93)-\mathrm{C}(88)-\mathrm{N}(9)$ & $121.6(11)$ & $C(109)-C(110)-C(115)$ & $120.1(13)$ \\
\hline $\mathrm{C}(88)-\mathrm{C}(89)-\mathrm{C}(90)$ & $119.7(13)$ & $C(112)-C(111)-C(110)$ & $121.7(17)$ \\
\hline $\mathrm{C}(88)-\mathrm{C}(89)-\mathrm{C}(94)$ & $121.9(12)$ & $C(111)-C(112)-C(113)$ & $119.6(16)$ \\
\hline C(90)-C(89)-C(94) & $118.3(11)$ & $C(111)-C(112)-C(116)$ & $120.3(16)$ \\
\hline C(91)-C(90)-C(89) & $120.0(12)$ & $C(113)-C(112)-C(116)$ & $120.1(14)$ \\
\hline $\mathrm{C}(90)-\mathrm{C}(91)-\mathrm{C}(92)$ & $117.6(14)$ & $C(112)-C(113)-C(114)$ & $121.4(13)$ \\
\hline
\end{tabular}




\begin{tabular}{|c|c|c|c|}
\hline $\mathrm{C}(109)-\mathrm{C}(114)-\mathrm{C}(113)$ & $117.6(12)$ & $\mathrm{C}(140)-\mathrm{C}(139)-\mathrm{C}(144)$ & $121.2(12)$ \\
\hline$C(109)-C(114)-C(117)$ & $117.6(11)$ & $\mathrm{C}(140)-\mathrm{C}(139)-\mathrm{N}(14)$ & $119.7(10)$ \\
\hline $\mathrm{C}(113)-\mathrm{C}(114)-\mathrm{C}(117)$ & $124.4(11)$ & $\mathrm{C}(144)-\mathrm{C}(139)-\mathrm{N}(14)$ & $119.0(12)$ \\
\hline $\mathrm{N}(12)-\mathrm{C}(118)-\mathrm{C}(123)$ & $119.2(12)$ & $C(141)-C(140)-C(139)$ & $117.9(11)$ \\
\hline $\mathrm{N}(12)-\mathrm{C}(118)-\mathrm{C}(119)$ & $120.9(13)$ & $C(141)-C(140)-C(145)$ & $119.4(13)$ \\
\hline$C(123)-C(118)-C(119)$ & $118.8(13)$ & $C(139)-C(140)-C(145)$ & $122.6(12)$ \\
\hline $\mathrm{C}(120)-\mathrm{C}(119)-\mathrm{C}(118)$ & $119.4(14)$ & $C(140)-C(141)-C(142)$ & $122.6(14)$ \\
\hline $\mathrm{C}(120)-\mathrm{C}(119)-\mathrm{C}(124)$ & $121.3(12)$ & $C(143)-C(142)-C(141)$ & $117.0(14)$ \\
\hline $\mathrm{C}(118)-\mathrm{C}(119)-\mathrm{C}(124)$ & $119.3(13)$ & $\mathrm{C}(143)-\mathrm{C}(142)-\mathrm{C}(146)$ & $123.9(13)$ \\
\hline $\mathrm{C}(119)-\mathrm{C}(120)-\mathrm{C}(121)$ & $123.0(15)$ & $\mathrm{C}(141)-\mathrm{C}(142)-\mathrm{C}(146)$ & $119.0(14)$ \\
\hline $\mathrm{C}(120)-\mathrm{C}(121)-\mathrm{C}(122)$ & $118.5(16)$ & $C(142)-C(143)-C(144)$ & $123.6(13)$ \\
\hline $\mathrm{C}(120)-\mathrm{C}(121)-\mathrm{C}(125)$ & $123.1(15)$ & $\mathrm{C}(143)-\mathrm{C}(144)-\mathrm{C}(139)$ & $117.5(13)$ \\
\hline $\mathrm{C}(122)-\mathrm{C}(121)-\mathrm{C}(125)$ & $118.3(16)$ & $C(143)-C(144)-C(147)$ & $119.3(11)$ \\
\hline $\mathrm{C}(123)-\mathrm{C}(122)-\mathrm{C}(121)$ & $120.8(16)$ & $\mathrm{C}(139)-\mathrm{C}(144)-\mathrm{C}(147)$ & $123.2(13)$ \\
\hline $\mathrm{C}(122)-\mathrm{C}(123)-\mathrm{C}(118)$ & $118.9(13)$ & $\mathrm{N}(16)-\mathrm{C}(148)-\mathrm{N}(15)$ & $110.6(12)$ \\
\hline $\mathrm{C}(122)-\mathrm{C}(123)-\mathrm{C}(126)$ & $118.9(14)$ & $\mathrm{N}(16)-\mathrm{C}(148)-\mathrm{S}(8)$ & $119.8(10)$ \\
\hline$C(118)-C(123)-C(126)$ & $122.1(13)$ & $\mathrm{N}(15)-\mathrm{C}(148)-\mathrm{S}(8)$ & $129.5(11)$ \\
\hline $\mathrm{N}(14)-\mathrm{C}(127)-\mathrm{N}(13)$ & $110.5(11)$ & $\mathrm{N}(15)-\mathrm{C}(149)-\mathrm{C}(150)$ & $108.9(13)$ \\
\hline $\mathrm{N}(14)-\mathrm{C}(127)-\mathrm{S}(7)$ & $131.9(10)$ & $\mathrm{N}(16)-\mathrm{C}(150)-\mathrm{C}(149)$ & $97.2(13)$ \\
\hline $\mathrm{N}(13)-\mathrm{C}(127)-\mathrm{S}(7)$ & $117.6(8)$ & $\mathrm{C}(156)-\mathrm{C}(151)-\mathrm{N}(15)$ & $123.7(12)$ \\
\hline $\mathrm{C}(129)-\mathrm{C}(128)-\mathrm{N}(13)$ & $98.6(10)$ & $\mathrm{C}(156)-\mathrm{C}(151)-\mathrm{C}(152)$ & $118.1(13)$ \\
\hline $\mathrm{N}(14)-\mathrm{C}(129)-\mathrm{C}(128)$ & $106.8(10)$ & $\mathrm{N}(15)-\mathrm{C}(151)-\mathrm{C}(152)$ & $118.0(13)$ \\
\hline $\mathrm{C}(131)-\mathrm{C}(130)-\mathrm{C}(135)$ & $122.3(13)$ & $C(153)-C(152)-C(151)$ & $119.2(14)$ \\
\hline $\mathrm{C}(131)-\mathrm{C}(130)-\mathrm{N}(13)$ & $123.0(12)$ & $\mathrm{C}(153)-\mathrm{C}(152)-\mathrm{C}(157)$ & $119.2(13)$ \\
\hline $\mathrm{C}(135)-\mathrm{C}(130)-\mathrm{N}(13)$ & $114.7(11)$ & $\mathrm{C}(151)-\mathrm{C}(152)-\mathrm{C}(157)$ & $121.6(14)$ \\
\hline $\mathrm{C}(130)-\mathrm{C}(131)-\mathrm{C}(132)$ & $121.4(13)$ & $C(154)-C(153)-C(152)$ & $120.7(15)$ \\
\hline $\mathrm{C}(130)-\mathrm{C}(131)-\mathrm{C}(136)$ & $118.5(12)$ & $\mathrm{C}(153)-\mathrm{C}(154)-\mathrm{C}(155)$ & $119.9(17)$ \\
\hline $\mathrm{C}(132)-\mathrm{C}(131)-\mathrm{C}(136)$ & $120.1(10)$ & $C(153)-C(154)-C(158)$ & $121.4(15)$ \\
\hline $\mathrm{C}(133)-\mathrm{C}(132)-\mathrm{C}(131)$ & $119.4(13)$ & $\mathrm{C}(155)-\mathrm{C}(154)-\mathrm{C}(158)$ & $118.7(17)$ \\
\hline $\mathrm{C}(132)-\mathrm{C}(133)-\mathrm{C}(134)$ & $120.0(14)$ & $C(156)-C(155)-C(154)$ & $119.0(16)$ \\
\hline $\mathrm{C}(132)-\mathrm{C}(133)-\mathrm{C}(137)$ & $119.8(13)$ & $C(155)-C(156)-C(151)$ & $123.0(13)$ \\
\hline $\mathrm{C}(134)-\mathrm{C}(133)-\mathrm{C}(137)$ & $120.1(14)$ & $C(155)-C(156)-C(159)$ & $118.7(14)$ \\
\hline $\mathrm{C}(133)-\mathrm{C}(134)-\mathrm{C}(135)$ & $120.2(14)$ & $\mathrm{C}(151)-\mathrm{C}(156)-\mathrm{C}(159)$ & $118.3(13)$ \\
\hline $\mathrm{C}(134)-\mathrm{C}(135)-\mathrm{C}(130)$ & $116.6(12)$ & $C(165)-C(160)-C(161)$ & $121.2(12)$ \\
\hline $\mathrm{C}(134)-\mathrm{C}(135)-\mathrm{C}(138)$ & $119.9(13)$ & $\mathrm{C}(165)-\mathrm{C}(160)-\mathrm{N}(16)$ & $121.3(12)$ \\
\hline $\mathrm{C}(130)-\mathrm{C}(135)-\mathrm{C}(138)$ & $123.3(13)$ & $\mathrm{C}(161)-\mathrm{C}(160)-\mathrm{N}(16)$ & $117.3(11)$ \\
\hline
\end{tabular}




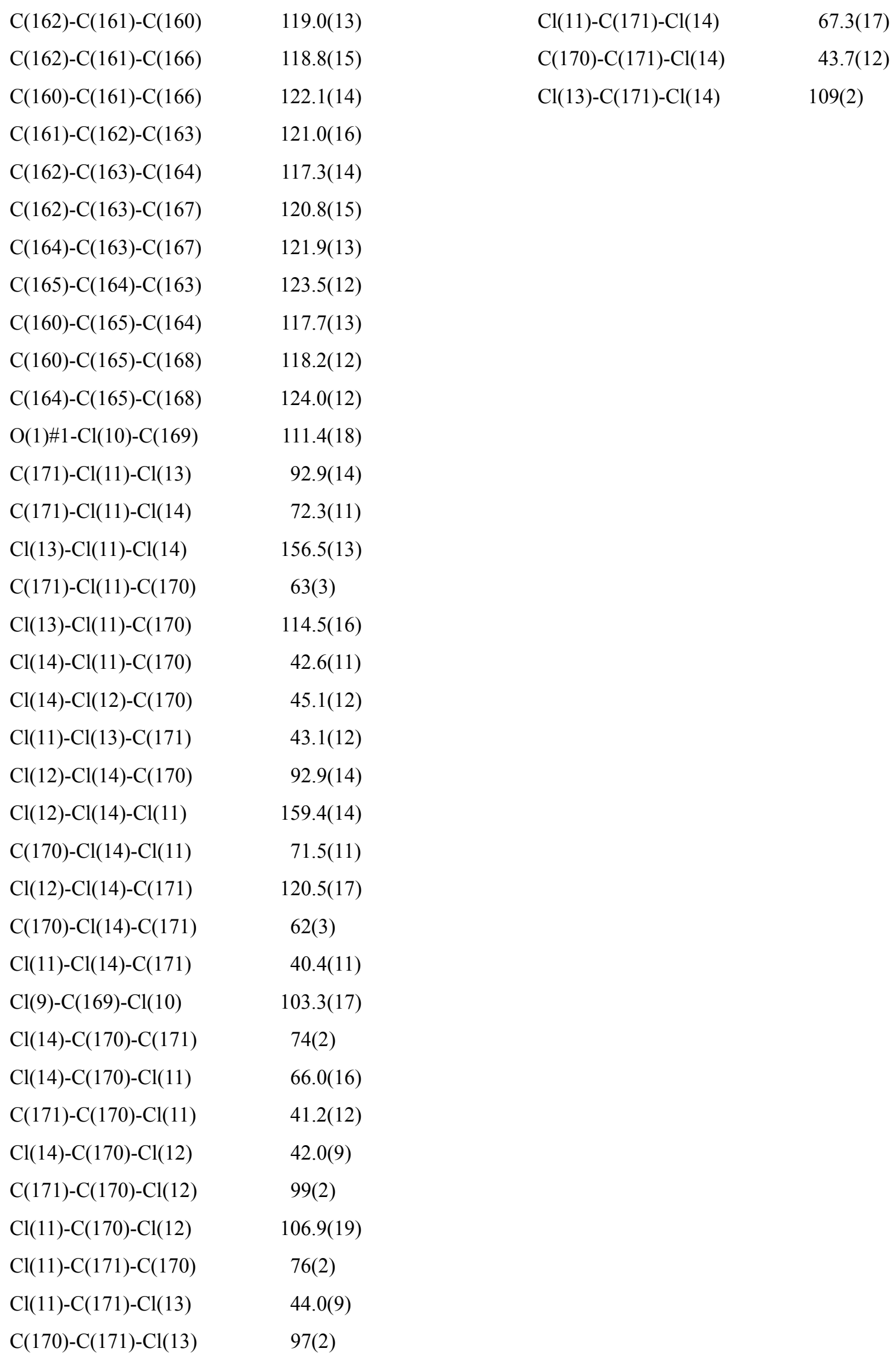


Symmetry transformations used to generate equivalent atoms:

$\# 1-\mathrm{x}+3 / 2, \mathrm{y}-1 / 2,-\mathrm{z} \quad \# 2-\mathrm{x}+3 / 2, \mathrm{y}+1 / 2,-\mathrm{z}$ 